\title{
Lorenz attractor through saddle-node bifurcations
}

\author{
by \\ C. A. MORALES \\ Instituto de Matematica Pura e Aplicada \\ Estrada Dona Castorina 110 \\ 22460 Rio de Janeiro, Brazil
}

\begin{abstract}
In this paper we consider the unfolding of a geometric Lorenz attractor when the singularity contained in this attractor goes through a saddle-node bifurcation. It is shown that these unfoldings can carry such a geometric Lorenz attractor either directly into a hyperbolic Plykin attractor or into phenomena associated to the unfolding of homoclinic tangencies like for instance strange Henon-like attractors and infinitely many sinks.
\end{abstract}

Key words: Lorenz attractors, Saddle-node Lorenz, Homoclinic tangencies.

RÉSUMÉ. - Nous considérons le déploiement d'un attracteur de Lorenz géométrique lorsque la singularité contenue dans l'attracteur passe par une bifurcation selle-nœud. On montre que ces déploiements contiennent un attracteur de Lorenz géométrique qui est soit dans un attracteur de Plykin hyperbolique ou bien associé à un phénomène de tangences homocliniques comme par exemple attracteurs étranges de type Hénon et infinité de puits.

\section{INTRODUCTION}

Here we will study some unfoldings of a geometric Lorenz attractor through a saddle-node bifurcation. We shall prove that these unfoldings can

1991 Mathematics Subject Classification. 58F14, 58F12, 58F13.

This work was supported by CNPq Brazil and CONICIT Venezuela. 
carry a geometric Lorenz attractor either into a Plykin attractor or, among other possibilities, into a strange Henon-like attractor or into a vector field with infinitely many sinks. These last two options are obtained through the creation of a homoclinic tangency. Let us start with a description of the main objects in these statements. Throughout the paper, an attractor for a vector field $X$ (a diffeomorphisms $f$ ) will be a transitive invariant set of $X$ (of $\mathrm{f}$ ) for which its basin of attraction has non empty interior.

A geometric Lorenz attractor is a vector field in $R^{3}$ which has an attractor with a dense set of hyperbolic periodic orbits and one hyperbolic singularity. Moreover, it is non-uniformly expanding and thus sensitive with respect to the initial conditions. Besides the classical Lorenz equations proposed by the author [8], for which the existence of a Lorenz attractor is still an unresolved question, the first concrete example of this kind of vector field was given by Guckemheimer and Williams (see [4]); more precisely, they exhibited an open set of vector fields in $R^{3}$ carrying a Lorenz attractor. A remarkable fact about these attractors is that they are persistent under every small perturbation of the vector field and yet they are nonhyperbolic. However, they do have some hyperbolic structure and positive Lyapunov exponent.

On the other hand, a Plykin attractor [12] will be for us the suspension (see [16]) of a diffeomorphism defined in a two-dimensional disc neighborhood which has a hyperbolic nontrivial attractor. We say that an attractor is nontrivial if it does not consist of a single periodic orbit. The existence of an open set of axiom A diffeomorphisms in the two-dimensional torus $T^{2}$ which contain at least one nontrivial attractor was proved by Smale [16]. The similar problem in the two dimensional disc or in $S^{2}$ remained unsolved until Plykin, who produced examples of open sets of axiom A diffeomorphisms in the two disc and in $S^{2}$ with nontrivial attractors.

Finally, by a Henon-like strange attractor we mean the suspension of a two-dimensional diffeomorphism which has an attractor $A$ with the following properties:

- it is nonhyperbolic.

- there is a saddle point $p \in A$ such that $A$ is the closure of the unstable manifold $W^{u}(p)$.

- there exists a dense orbit in $A$ which has positive Lyapunov exponent.

It is well known that these attractors appear in the unfolding of a homoclinic tangency (see [9]). Also appears with such an unfolding, the so-called infinitely many sinks phenomenum which consists of an interval, in the parameter space, in which there exists a residual set of points 
corresponding to vector fields with infinitely many coexisting attracting periodic orbits (sink type).

The present study of the unfolding of a Lorenz attractor through a saddlenode bifurcation was motivated by [3], where they considered the unfolding of surfaces diffeomorphisms that appears when a hyperbolic (saddle type) set $H$ goes through a saddle-node bifurcation by collapsing a sink and a saddle whose stable and unstable manifolds bound the hyperbolic set $H$. In this last reference it was proved that, in this case, hyperbolic parameters (i.e. parameters which correspond to a hyperbolic diffeomorphism) and Henon-like strange attractor parameters are prevalent phenomena, in other words, they have positive density at the initial bifurcating parameter value.

In order to study a similar situation for a three dimensional vector field with a Lorenz attractor, we introduce a class of vector fields which we call Saddle-node Lorenz attractors. It is defined as follows:

A Saddle-node Lorenz attractor will be a three-dimensional vector field for which its nonwandering set consists of a hyperbolic set together with an attractor in which there exists a dense set of periodic hyperbolic orbits and at least one saddle-node singularity.

These Saddle-node Lorenz attractors are obtained when the singularity contained in a geometric Lorenz attractor goes through a saddle-node bifurcation by collapsing it with another saddle singularity in a similar way as that in the case of hyperbolic sets for surface diffeomorphisms mentioned before.

The main purpose of this paper is to study some of what we have called Saddle-node Lorenz attractors as specified in the sequel. We introduce here two definitions:

Definition. - We say that a one-parameter family $Y=\left\{Y_{\mu}\right\}_{\mu \in[-1,1]}$ unfolds a Lorenz attractor in a homoclinic tangency iff there exists a parameter value $\mu_{b} \in(-1,1)$ and a $\delta>0$ such that the following hold:

a) if $\mu \in\left(\mu_{b}-\delta, \mu_{b}\right)$, then $Y_{\mu}$ have a Lorenz attractor and there are no homoclinic nor heteroclinic tangencies associated to hyperbolic periodic orbits of $Y_{\mu}$;

b) $Y_{\mu_{b}}$ is a Saddle-node Lorenz and there are no homoclinic nor heteroclinic tangencies associated to hyperbolic periodic orbits of $Y_{\mu_{b}}$;

c) there is a parameter sequence $\left(\mu_{k}\right) \rightarrow \mu_{b}^{+}$, such that every $Y_{\mu_{k}}$ unfolds a homoclinic tangency associated to some hyperbolic saddle-type periodic orbit;

d) there is no parameter value $\mu \in\left(\mu_{b}, \mu_{b}+\delta\right)$ for which $Y_{\mu}$ has a Lorenz attractor. 
Also we say that the family $\mathrm{Y}$ unfolds a Lorenz attractor directly into a Plykin attractor iff there exist $\mu_{b} \in(-1,1)$ and $\delta>0$, for which the following holds:

a') if $\mu \in\left(\mu_{b}-\delta, \mu_{b}\right)$, then $Y_{\mu}$ has a Lorenz attractor and no Plykin attractors. In addition, there are no homoclinic nor heteroclinic tangencies associated to hyperbolic periodic orbits of $Y_{\mu}$;

b') $Y_{\mu_{b}}$ is a Saddle-node Lorenz vector field with no Plykin attractors. Also, there are no homoclinic nor heteroclinic tangencies associated to hyperbolic periodic orbits of $Y_{\mu_{b}}$;

c') if $\mu \in\left(\mu_{b}^{\prime}, \mu_{b}+\delta\right)$, then $Y_{\mu}$ is an axiom A (see [14]) with a single Plykin attractor.

With these two definitions in mind we shall give the statements of two Theorems which describe the unfolding of some Saddle-node Lorenz attractors. The first one implies that Henon-like strange attractors and vector fields with infinitely many coexisting sinks can accumulate certain class of Saddle-node Lorenz vector fields because of well known properties about generic unfoldings of a homoclinic tangency associated to a hyperbolic periodic orbit (see [9] or [14]). The second one says that one can separate the set of geometric Lorenz attractors and those vector fields carrying a Plykin attractor by a codimension one submanifold. This result is directly related with the bifurcation mechanism showed by Afraimovic and Shilnikov [1] (see also [17]), where the authors proved that this kind of separation can occur between the Morse-Smale systems and those with a countable number of hyperbolic periodic orbits. It is interesting to point out that the Theorem II below also implies an approximation, by Plykin attractors, of some of the Saddle-node Lorenz attractors showed here. Such an approximation by Plykin attractors holds, for instance, when the initial flow is a constant vector field on the three dimensional torus (see [11]).

THEOREM I. - There exists an open set $U$ in the space of one-parameter families of vector fields in $R^{3}$ for which there exists a residual subset $U$ ' in $U$, such that every $Y \in U$ ' unfolds a Lorenz attractor in a homoclinic tangency.

THEOREM II. - There exists an open set $W$ in the space of one-parameter families of vector fields in $R^{3}$ for which every $Y \in W$ unfolds a Lorenz attractor directly into a Plykin attractor.

Let us now present a question, that we believe it is very interesting. In [3], generic one-parameter unfolding of critical saddle-node cycles for surface diffeomorphisms were considered in the context of strange attractors. It was shown that such a bifurcating diffeomorphism is a positive Lebesgue density point, in the parameter line, for Henon-like attractors. Our question here concernes the possibility of obtaining a similar result, when considering 
two-parameter unfoldings of the above dynamic configurations exhibiting a cycle of saddle-node singularities. Similarly, we can ask about the density of hyperbolic systems (or even total density) in this context. These questions will be addressed by the author somewhere else.

This article is organized as follows: in $\S 2$ we give some preliminar concepts and start the proof of both Theorems by studying the unfolding of some Saddle-node Lorenz attractors. These will be obtained in a similar way that the ones given in [4]. In $\S 3$, we construct the open set $U$ in order to prove the Theorem I. Finally, in $\S 4$ we will construct the open set W in order to prove the Theorem II.

\section{SADDLE-NODE LORENZ ATTRACTORS}

In this section a initial vector field $X_{0}$ is introduced. It will be a Saddlenode Lorenz attractor in the sense of the definition given in $\S 1$ and its contruction will be similar to the one given in [4]. In what follows $W^{s}$, $W^{u}, W^{c s}, W^{c u}$ will denote the classical invariant manifolds associated to either a singularity or a periodic orbit of a given vector field, namely the stable, unstable, centre stable and centre unstable manifolds respect. Also, we denote by $f_{x}$ the derivative of a given function $f$ with respect to the spacial coordinate $x$.

The vector field $X_{0}$ will satisfy the following hypotheses:

H-1) $X_{0}$ is, in a fixed neighborhood of $(0,0,0)$, as follows

$$
\left\{\begin{array}{l}
\dot{x}=\lambda_{1} \cdot x \\
\dot{y}=-\lambda_{2} \cdot y \\
\dot{z}=-z^{2} \quad \lambda_{i}>0, \lambda_{1}<\lambda_{2}
\end{array}\right.
$$

This neighborhood will be a small one of the cube $[-1,1]^{3}$. We notice that $(0,0,0)$ is a saddle-node singularity of $X_{0}$.

Let $\mathrm{S}$ be the square $[-1 / 2,1 / 2]^{2} \times\{1\}$. If $\left(x_{0}, y_{0}, 1\right)$ lies in $\mathrm{S}$, we can solve $X_{0}$ to obtain the solution:

$$
\left\{\begin{array}{l}
x=x_{0} \cdot e^{\lambda_{1} \cdot t} \\
y=y_{0} \cdot e^{-\lambda_{2} \cdot t} \\
z=\frac{1}{1+t} \quad t \geq 0
\end{array}\right.
$$

Set $S^{*}=\mathrm{S} /\left\{x_{0}=0\right\}=S^{+} \cup S^{-}$. Then a flow-defined map $\pi_{l o c, 0}: S^{*} \rightarrow$ $\{x= \pm 1\}$ is defined and it has the following form

$$
\pi_{l o c, 0}:\left(x_{0}, y_{0}, 1\right) \longrightarrow\left(\operatorname{sig}\left(x_{0}\right), y_{0} \cdot\left|x_{0}\right|^{\frac{\lambda_{2}}{\lambda_{1}}}, \frac{1}{1-\left(1 / \lambda_{1}\right) \log \left|x_{0}\right|}\right)
$$


so it carries $S^{*}$ into two cusp triangle as that in the picture (e) below

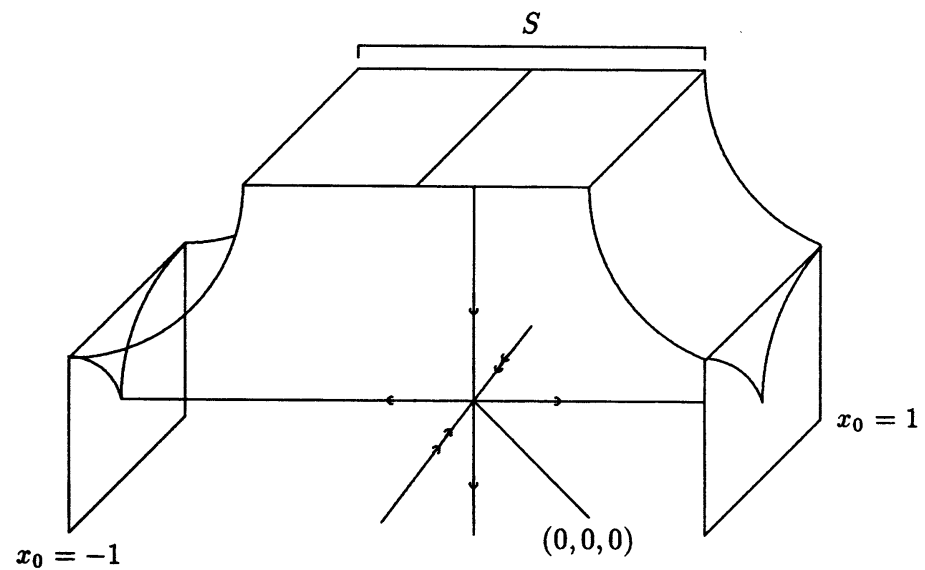

(e)

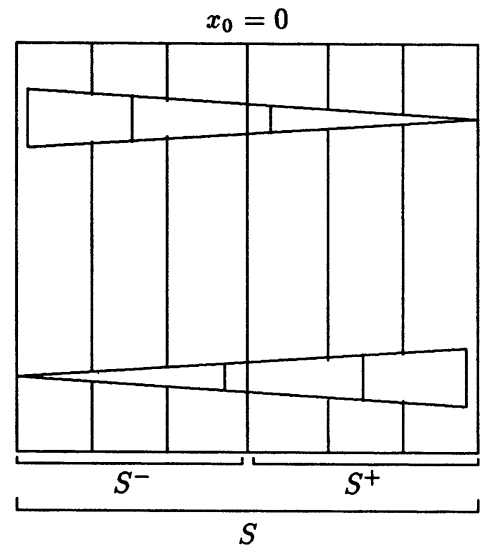

(f)

Fig. 1 .

H-2) The $X_{0}$ 's flow carries both cusp triangles back into $\mathrm{S}$ in such a way that we have a first return map $\pi_{0}$ defined in $S^{*}$. It sends $S^{*}$ into $\mathrm{S}$. We assume that it has the following form

$$
\pi_{0}\left(x_{0}, y_{0}\right)=\left(f_{0}\left(x_{0}\right), g_{0}\left(x_{0}, y_{0}\right)\right)
$$


with

$$
\begin{cases}\text { a) } & g_{0}\left(x_{0}, y_{0}\right)>1 / 4, x_{0}>0,\left|g_{0, y_{0}}\right| \text { small } \\ \text { b) } g_{0}\left(x_{0}, y_{0}\right)<1 / 4, x_{0}<0 \\ \text { c) } f_{0}\left(0^{+}\right)=-1 / 2 \quad f_{0}\left(0^{-}\right)=1 / 2 \\ \text { d) } f^{\prime}(x)>\sqrt{2}-1 / 2<f(x)<1 / 2\end{cases}
$$

Therefore, the vertical foliation is preserved by $\pi_{0}$. Besides this property, it is also contracted by this map (see picture (f) above).

Remarks.

1) That construction is quite similar to the one given in [4]. The difference now is that the singularity involved is a saddle-node singularity.

2) The arguments given in [4] imply that $X_{0}$ has an attractor with periodic orbits and the saddle-node $(0,0,0)$. Furthermore, $X_{0}$ is a Saddle-node Lorenz vector field.

Now, let $X=\left\{X_{\mu}\right\}_{\mu \in[-1,1]}$ be a one-parameter family which has the following form (in the neighborhood which comes from (H-1))

$$
\left\{\begin{array}{l}
\dot{x}=\lambda_{1} \cdot x \\
\dot{y}=-\lambda_{2} \cdot y \\
\dot{z}=-\mu-a . z^{2}
\end{array}\right.
$$

We are interested in families $Y=\left\{Y_{\mu}\right\}_{\mu \in[-1,1]}$ close to $\mathrm{X}$. To study them we have the next Proposition

Proposition 2.1. - Suppose that $X$ is generic. If $Y$ is closed enough to $X$, then there exists a parameter value $\mu_{b} \in(-1,1)$ close to zero such that $Y_{\mu}$ has a Lorenz attractor if $\mu<\mu_{b}$. In addition, $Y_{\mu_{b}}$ is Saddle-node Lorenz vector field.

Among another facts, this Proposition will imply that, under generic conditions, the Saddle-node Lorenz vector fields belong to a codimension one submanifold. Using a theorem due to Takens [19], which is also used in [13], we will give the exact meaning of "generic" in the Proposition 2.1.

THEOREM 2.2. - Suppose that the eigenvalues of $X_{0}$ satisfies certain open and dense Stermberg conditions (see [13]). Then for families $Y=\left\{Y_{\mu}\right\}_{\mu \in[-1,1]}$ near $X$ there exists a parameter value $\mu_{b} \in(-1,1)$ such that $Y_{\mu}$ is $C^{2}$-conjugate to the following vector field

$$
\left\{\begin{array}{l}
\dot{x}=\lambda_{1} \cdot x+f(z, \mu) \cdot x \\
\dot{y}=-\lambda_{2} \cdot y+g(z, \mu) \cdot y \\
\dot{z}=-b \cdot\left(\mu-\mu_{b}\right)-a \cdot z^{2}+h(z, \mu)=H(z, \mu)
\end{array}\right.
$$

where $b$ and $a$ are close to one. Also the functions $f, g, h$ are $C^{2}$-close to the zero function and $h\left(0, \mu_{b}\right)=h_{z}\left(0, \mu_{b}\right)=h_{\mu}\left(0, \mu_{b}\right)=h_{z z}\left(0, \mu_{b}\right)=0$. The conjugacy depends continuously on the family $Y$. 
We notice that in [4] some diophantine conditions on the eigenvalues were used to obtain $C^{1}$-linearization of the singularities, to make the computations in that work easier. Certainly, the same observation applies to the present paper.

Hereafter we will assume that the parameter $\mu_{b}$ is zero for $\operatorname{arcs} Y$ close to $X$ since the saddle-node singularity is a codimension one phenomenum (see [18]).

In order to prove the Proposition 2.1 we assume that $Y$ is as that in (2) with $\mu_{b}=0$. Let us denote by $\pi_{\mu}$ the first return map, defined in $S^{*}$, associated to $Y_{\mu}$. Therefore, $Y$ induce a one-parameter family of maps $\left\{\pi_{\mu}\right\}_{\mu \in[-1,1]}$. These are discontinuous for $\mu \leq 0$ and diffeomorphisms for $\mu>0$, as we will see in the sequel. Our goal here is to study these maps when $\mu \leq 0$. As first step, we shall prove the existence of a $C^{1}$ strong stable, $\pi_{\mu}$-invariant foliation defined in the whole set $S$ with the line $\left\{x_{0}=0\right\}$ as leave. This will let us to reduce the dynamics of the vector field to that of a one dimensional map. It will be a expansive map and so the approach given in [4] can be applied in order to get the existence of a transitive attracting set with hyperbolic periodic orbits and, at least, one hyperbolic saddle singularity.

If we consider the system (2) (with $\mu_{b}=0$ ), we can see that for values $\mu<0$ there exist two singularities $\left(0,0, z_{ \pm}(\mu)\right)=E_{ \pm}(\mu)$ which are hyperbolic with index 1 and 2 respect. Also $z_{+}(\mu)>0>z_{-}(\mu)$ and $\left|z_{+}(\mu)\right|$ and $\left|z_{-}(\mu)\right|$ are $O\left(\sqrt{-\frac{b \cdot \mu}{a}}\right)$ for negative parameter values $\mu$.

Take $\left(x_{0}, y_{0}, 1\right)$ in $\mathrm{S}$ and the solution of $(2)$ with such a initial condition. Denote it by $\left(x\left(t, x_{0}, \mu\right), y\left(t, y_{0}, \mu\right), z(t, \mu)\right)$. Then, we have the two following functional equations:

$$
\left\{\begin{array}{l}
x(t, \mu)=x_{0} \cdot e^{\left[\lambda_{1} \cdot t+\int_{0}^{t} f(z(s, \mu), \mu) d s\right]} \\
y(t, \mu)=y_{0} \cdot e^{\left[-\lambda_{2} \cdot t+\int_{0}^{t} g(z(s, \mu), \mu) d s\right]}
\end{array}\right.
$$

The above considered map $\pi_{\mu}$ is then obtained by the composition of two maps, namely the local map $\left(\pi_{l o c, \mu}\right)$ and the global map $\left(\pi_{f a r, \mu}\right)$. The first one is defined as follows

$$
\begin{aligned}
\pi_{l o c, \mu}: S^{*} & \longrightarrow\{x=1, x=-1\} \\
\pi_{l o c, \mu}\left(x_{0}, y_{0}\right) & =\left(y\left(\tau_{0}, \mu\right), z\left(\tau_{0}, \mu\right)\right)
\end{aligned}
$$

where $\tau_{0}$ is defined by the implicit equation in $t$ given by

$$
x\left(t, x_{0}, y_{0}, \mu\right)= \pm 1
$$


this map is represented in the picture below

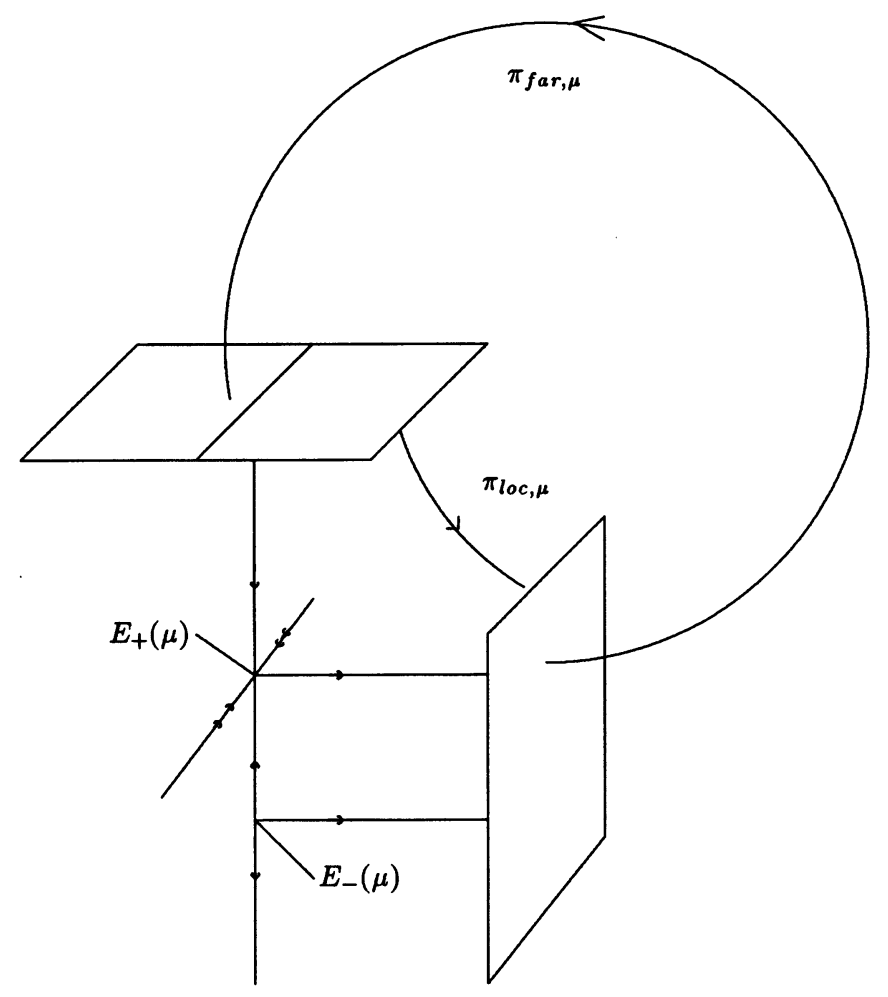

Fig. 2.

The second map is related to the global behavior of the flow. It carries $\{x= \pm 1\}$ back into $\mathrm{S}$ as above.

The derivative of $\pi_{f a r, \mu}$ has the form

$$
\left(\begin{array}{ll}
\tilde{a}_{\mu} & \tilde{b}_{\mu} \\
\tilde{c}_{\mu} & \tilde{d}_{\mu}
\end{array}\right)
$$

with $\tilde{a}_{0}=\tilde{d}_{0}=0,\left|\tilde{b}_{0}\right|$ bounded away fron zero and $\tilde{c}_{0}$ small enough.

We shall prove the following Proposition which yields the proof of Proposition 2.1.

Proposition 2.3. - For $\left\{Y_{\mu}\right\}_{\mu \in[-1,1]}$ as that in (2) (with $\mu_{b}=0$ ) and for nonpositive parameter values $\mu$ close to zero, there exists a $C^{1}$-invariant foliation for $\pi_{\mu}=\pi_{l o c, \mu} \circ \pi_{f a r, \mu}$. Moreover, it is contractive and almost Vol. $13, \mathrm{n}^{\circ}$ 5-1996. 
vertical. Also, the one dimensional map $f_{\mu}$ associated to this foliation is locally eventually onto (see [4]).

The proof of the Proposition 2.3 goes through the next lemmas.

LEMMA 2.4. - For every $\epsilon>0$

$$
a-\epsilon \leq-\frac{H(z, \mu)}{z^{2}-z_{+}^{2}(\mu)} \leq a+\epsilon
$$

if $\mu$ is negative, close to zero and $h$ is small enough (recall (2)).

Proof. - We set

$$
H(z, \mu)=-a\left(z^{2}-z_{+}^{2}(\mu)\right)+h(z, \mu)-h\left(z_{+}(\mu), \mu\right)
$$

then

$$
-\frac{H(z, \mu)}{z^{2}-z_{+}^{2}(\mu)}=a-\left\{\frac{h_{z}\left(z_{+}(\mu), \mu\right)}{z+z_{+}(\mu)}+\frac{R(z, \mu)}{z^{2}-z_{+}^{2}(\mu)}\right\}
$$

where $R$ is a function such that $R\left(z_{+}(\mu), \mu\right)=R_{z}\left(z_{+}(\mu), \mu\right)=0$ for all $\mu$. But

$$
\left|\frac{h_{z}\left(z_{+}(\mu), \mu\right)}{z+z_{+}(\mu)}\right| \leq \frac{\mu}{2 . z_{+}(\mu)}+O\left(z_{+}(\mu)+\left(\mu^{2} / z_{+}(\mu)\right)\right)<\epsilon / 2
$$

if $\mu$ is small enough. On the other hand

$$
\left|\frac{R(z, \mu)}{z^{2}-z_{+}^{2}(\mu)}\right| \leq\left|h_{z z}(\eta, \mu)\right| \cdot\left|\frac{z-z_{+}(\mu)}{z+z_{+}(\mu)}\right|
$$

where $\eta$ lies between $z$ and $z_{+}(\mu)$. Hence, we are done if $h$ is close to zero because

$\left|z-z_{+}(\mu)\right|<\left|z+z_{+}(\mu)\right|$ for $\mu<0$ small.

LEMMA 2.5. - Let $\alpha$ be a positive, fixed number. Let us define, for any $\Delta>0$ and $\mu<0$ :

$$
\rho_{\mu}(\Delta)=\left\{\begin{array}{l}
\inf _{z \in\left(z_{+}(\mu), \Delta\right]} \mid H(z, \mu) \cdot e^{-\alpha \int_{z}^{1} \frac{d s}{H(s, \mu)} \mid} ; \text { if } z_{+}(\mu)<\Delta \\
0 \quad \text { otherwise }
\end{array}\right.
$$

then, for every real number $M$ there exists $\Delta(M)$ positive such that for any $0<\Delta<\Delta(M), \rho_{\mu}(\Delta)>M$ if $\mu$ is negative, close to zero.

Proof. - Using Lemma 2.4 it follows that

$$
\alpha(a-\epsilon) \int_{z}^{1} \frac{d s}{s^{2}-z_{+}^{2}(\mu)} \leq-\alpha \int_{z}^{1} \frac{d s}{H(s, \mu)} \leq \alpha(a+\epsilon) \int_{z}^{1} \frac{d s}{s^{2}-z_{+}^{2}(\mu)}
$$


if $z \in\left(z_{+}(\mu), 1\right]$. Now

$$
e^{\left[\int_{z}^{1} \frac{d s}{s^{2}-z_{+}^{2}(\mu)}\right]}=\left\{\frac{\left(z+z_{+}(\mu)\right)\left(1-z_{+}(\mu)\right)}{\left(z-z_{+}(\mu)\right)\left(1+z_{+}(\mu)\right)}\right\}^{\left[1 / 2 z_{+}(\mu)\right]}
$$

by a direct computation. Then, all we need to prove is that the following number

$$
B=\left(z^{2}-z_{+}^{2}(\mu)\right)\left\{\frac{\left(z+z_{+}(\mu)\right)\left(1-z_{+}(\mu)\right)}{\left(z-z_{+}(\mu)\right)\left(1+z_{+}(\mu)\right)}\right\}^{\left[1 / 2 z_{+}(\mu)\right]}
$$

is large for every $z$ in an appropriate domain. Let us define, for $x>\beta>0$

$$
f(x, \beta)=\frac{(x+\beta)^{[1 / 2 \beta+1]}}{(x-\beta)^{[1 / 2 \beta-1]}}
$$

Claim

For every $M>0$ there exists $\beta_{0}>0$ and $\Delta_{0}>\beta_{0}$ such that

$$
f(x, \beta)>M
$$

if $0<\beta<\beta_{0}$ and $\beta<x<\Delta_{0}$.

This claim clearly implies the Lemma 2.5. Now, to prove it we first observe that $f_{x}(x, \beta)<0$ if $0<x<1 / 2$. Hence it follows that

$$
f(x, \beta) \geq \frac{(1 / 2 N+\beta)^{[1 / 2 \beta+1]}}{(1 / 2 N-\beta)^{[1 / 2 \beta-1]}}
$$

if $\beta<x<1 / 2 N$, with $\beta$ small and $N$ being a fixed, big number. Therefore

$$
f(x, \beta) \geq \frac{(1+2 \beta N)^{[1 / 2 \beta+1]}}{(1-2 \beta N)^{[1 / 2 \beta-1]}} \cdot(2 N)^{-2}
$$

when $\beta<x<1 / 2 N$. But $\frac{(1+2 \beta N)^{[1 / 2 \beta+1]}}{(1-2 \beta N)^{[1 / 2 \beta-1]}}$ goes to $e^{2 N}$ if $\beta$ goes to zero and $e^{2 N}(2 N)^{-2}$ is large if $N$ is large enough. This finishes the proof of the claim.

This last Lemma implies the following Corollary. Recall the definitions of $y\left(t, y_{0}, \mu\right), z(t, \mu)$ and $\tau_{0}$ given above.

COROLlaRY 2.6. - There exist $K>0$ and $\alpha>0$ such that

$$
\left|\frac{\dot{y}\left(t, y_{0}, \mu\right)}{\dot{z}(t, \mu)}\right| \leq M e^{-\alpha t}
$$

if $t \in\left[0, \tau_{0}\right]$.

Vol. $13, \mathrm{n}^{\circ}$ 5-1996. 
Now, the next Proposition give a version of the Proposition of the section II in [15] for generic unfolding of a saddle-node singularity. We use the notation $\dot{z}$ for $\dot{z}\left(\tau_{0}, \mu\right), \tau_{x_{0}}$ for $\tau_{0, x_{0}}\left(x_{0}, \mu\right)$ and so on. Recall that $\tilde{a}_{\mu}, \tilde{b}_{\mu}, \tilde{c}_{\mu}$ and $\tilde{d}_{\mu}$ are the entries of the matrix $D \pi_{f a r, \mu}$.

Proposition 2.7. - Suppose $\mu<0$. Then for the matrix $D \pi_{\mu}\left(x_{0}, y_{0}\right)=$ $\left(\begin{array}{ll}A_{\mu} & B_{\mu} \\ C_{\mu} & D_{\mu}\end{array}\right)$ the following properties hold

$$
\begin{cases}A_{\mu}\left(x_{0}, y_{0}\right)=K_{1, \mu}\left(x_{0}, y_{0}\right) \cdot \tau_{x_{0}} \cdot \dot{z} & C_{\mu}\left(x_{0}, y_{0}\right)=K_{3, \mu}\left(x_{0}, y_{0}\right) \cdot \tau_{x_{0}} \cdot \dot{z} \\ B_{\mu}\left(x_{0}, y_{0}\right)=K_{2, \mu}\left(x_{0}, y_{0}\right) \cdot y_{y_{0}} & D_{\mu}\left(x_{0}, y_{0}\right)=K_{4, \mu}\left(x_{0}, y_{0}\right) \cdot y_{y_{0}}\end{cases}
$$

and there exist constants $K^{\prime}, K^{\prime \prime}>0$ such that $\left|K_{i, \mu}\right| \leq K^{\prime \prime} i=1,2,3,4$; $\left|K_{1, \mu}\right| \geq K^{\prime}$. Also the quotient $\left|\frac{K_{2, \mu}}{K_{1, \mu}}\right|,\left|\frac{K_{3, \mu}}{K_{1, \mu}}\right|$ and $\left|\frac{K_{4, \mu}}{K_{1, \mu}}\right|$ are small.

Proof. - We can see that $z_{x_{0}}=0, y_{x_{0}}=0$ and $\tau_{y_{0}}=0$. Hence we have the next equalities

$$
\left\{\begin{array}{l}
A_{\mu}=\left(\tilde{a}_{\mu} \cdot \dot{y}+\tilde{b}_{\mu} \cdot \dot{z}\right) \cdot \tau_{x_{0}} ; \quad C_{\mu}=\left(\tilde{c}_{\mu} \cdot \dot{y}+\tilde{d}_{\mu} \cdot \dot{z}\right) \cdot \tau_{x_{0}} \\
B_{\mu}=\tilde{a}_{\mu} \cdot y_{y_{0}} \quad ; \quad D_{\mu}=\tilde{c}_{\mu} \cdot y_{y_{0}}
\end{array}\right.
$$

Now we can take $K_{1, \mu}=\tilde{a}_{\mu} \cdot(\dot{y} / \dot{z})+\tilde{b}_{\mu}, K_{2, \mu}=\tilde{a}_{\mu}, K_{3, \mu}=\tilde{c}_{\mu} \cdot(\dot{y} / \dot{z})+\tilde{d}_{\mu}$ and $K_{4, \mu}=\tilde{c}_{\mu}$. Next, we observe that $K_{1, \mu} \approx \tilde{b}_{\mu}$. So it is bounded away from zero and also $\tilde{d}_{\mu}, \tilde{a}_{\mu}$ and $\tilde{c}_{\mu}$ are small. This finishes the proof of the Proposition 2.7.

Proof of the Proposition 2.3. - If $(\varphi, 1)$ is a vector such that $|\varphi|<\epsilon$ (where $\epsilon$ is small), then $D \pi_{\mu}^{-1}(\varphi, 1)$ is a vector with slope

$$
|T(\varphi)|=\left|\frac{D_{\mu} \cdot\left(\varphi \circ \pi_{\mu}\right)-B_{\mu}}{A_{\mu}-C_{\mu} \cdot\left(\varphi \circ \pi_{\mu}\right)}\right| \leq \frac{\left|D_{\mu} / A_{\mu}\right| \cdot|\varphi|+\left|B_{\mu} / A_{\mu}\right|}{1-\left|C_{\mu} / A_{\mu}\right| \cdot|\varphi|}
$$

now, the Proposition 2.7 yields $\left|D_{\mu} / A_{\mu}\right|,\left|B_{\mu} / A_{\mu}\right|<\rho .\left|y_{y_{0}} / \dot{z} \tau_{x_{0}}\right|$ and $\left|C_{\mu} / A_{\mu}\right|<\rho$ with $\rho$ positive and small. But $\left|y_{y_{0}}\right|$ is about $e^{-\alpha \tau_{0}}$ for some constant $\alpha>0$ because the functional equations (2) apply with $g$ small. Also, these equations give us that $\tau_{x_{0}}$ is about $x_{0}^{-\beta}$ for some another constant $\beta>0$. Therefore $\left|y_{y_{0}} / \dot{z} \tau_{x_{0}}\right|$ is bounded and then it follows that

$$
|T(\varphi)|<\epsilon
$$

if $|\varphi|<\epsilon$, where $|$.$| means here supremun norm. Thus T$ defines an operator on the space $\{|\varphi|<\epsilon\}$. Moreover, $T$ is a contraction because of the following computation

$$
\left|\frac{D_{\mu}\left(\varphi \circ \pi_{\mu}\right)-B_{\mu}}{A_{\mu}-C_{\mu}\left(\varphi \circ \pi_{\mu}\right)}-\frac{D_{\mu}\left(\varphi^{*} \circ \pi_{\mu}\right)-B_{\mu}}{A_{\mu}-C_{\mu}\left(\varphi^{*} \circ \pi_{\mu}\right)}\right| \leq \frac{\rho(1+\rho)}{(1-\rho \epsilon)^{2}} \cdot\left|\varphi-\varphi^{*}\right| \leq \frac{1}{2} \cdot\left|\varphi-\varphi^{*}\right|
$$


if $\rho$ and $\epsilon$ are small enough. Therefore, the Contracting Map Theorem (see [5]) applies to obtain a fixed point $\varphi_{0}$ of $T$ which induce a $C^{0}$ invariant, contractive and almost vertical foliation for $\pi_{\mu}$. Now we prove that $\varphi_{0}$ is a $C^{1}$-map. First, we look at the following operator

$$
\begin{aligned}
S(\varphi) & (\mathcal{A}) \\
= & \left\{\left[A_{\mu}-C_{\mu}\left(\varphi \circ \pi_{\mu}\right)\right]\left[\left(\varphi \circ \pi_{\mu}\right) \cdot \nabla D_{\mu}-\nabla B_{\mu}\right]\right. \\
& \left.-\left[D_{\mu}\left(\varphi \circ \pi_{\mu}\right)-B_{\mu}\right] \cdot\left[\nabla A_{\mu}-\left(\varphi \circ \pi_{\mu}\right) \nabla C_{\mu}\right]\right\} \\
& .\left[A_{\mu}-C_{\mu}\left(\varphi \circ \pi_{\mu}\right)\right]^{-2}+\frac{\operatorname{det} D \pi_{\mu}}{\left[A_{\mu}-C_{\mu}\left(\varphi \circ \pi_{\mu}\right)\right]^{2}} \cdot\left(\mathcal{A} \circ \pi_{\mu}\right) \cdot D \pi_{\mu}
\end{aligned}
$$

(see [6] or [2]), where $\nabla$ is the classical gradient function and $D$ is the total derivative, with respect to $\left(x_{0}, y_{0}\right)$. Actually, we have to study the following map

$$
(\varphi, \mathcal{A}) \longrightarrow(T(\varphi), S(\varphi)(\mathcal{A}))
$$

which is defined for $(\varphi, \mathcal{A})$ in a appropriate functional space and we shall prove that the map $\mathcal{A} \rightarrow S(\varphi)(\mathcal{A})$ is a well defined uniform contraction for every $\varphi$ (see also [15]). We get it by looking at three facts which follows by using the Lemma 2.4, Lemma 2.5 and the Corollary 2.6: if $w$ is the map defined as $w\left(x_{0}, y_{0}, \mu\right)=y_{y_{0}} / \dot{z} \tau_{x_{0}}$ then

1) $\nabla w\left(x_{0}, y_{0}, \mu\right)$ has a continuous extension to $x_{0}=0$ by setting $\nabla w\left(0, y_{0}, \mu\right)=0$

2) It can be proved that $w\left(x_{0}, y_{0}, \mu\right) . \nabla K_{i, \mu}\left(x_{0}, y_{0}, \mu\right) \rightarrow 0$ when $x_{0} \rightarrow 0$.

3) The coefficient of $\mathrm{A}$ in the equation of $S(\varphi)(\mathcal{A})$ is small by checking the calculations which was done before.

Now, we show that the one dimensional map induced by $\pi_{\mu}$ in the foliation given by $\varphi_{0}$ is locally eventually onto. It can be done by seeing that near $x_{0}=0$ the derivative of the induced map $f_{\mu}$ is large and therefore it is also large in its domain by continuity off side a fixed neighborhood of $x_{0}=0$. Hence $f_{\mu}{ }^{\prime}>\sqrt{2}$ and then by [4] it is a locally eventually onto map. Finally we can prove that $Y_{0}$ is a Saddle-node Lorenz by doing a similar approach as the above descripted, indeed we shall obtain the corresponding version of the Lemma 2.5, Corollary 2.6 and the Proposition 2.7 and then proceed as before. This finishes the proof of the Proposition 2.3 and the Proposition 2.1.

\section{PROOF OF THE THEOREM I}

Our goal here is to prove the Theorem I. For this purpose we construct a initial one-parameter family $X=\left\{X_{\mu}\right\}_{\mu \in[-1,1]}$ for which H-1, H-2 and Vol. 13, $\mathrm{n}^{\circ}$ 5-1996. 
equation (1) of $\S 2$ hold, so the conclutions given above apply in order to obtain that for nearby arcs $Y=\left\{Y_{\mu}\right\}_{\mu \in[-1,1]} Y_{\mu}$ is a Lorenz attractor if $\mu<\mu_{b}$ and $Y_{\mu_{b}}$ is a Saddle-node Lorenz vector field, where $\mu_{b}$ comes from the Proposition 2.1. Hence the Theorem I will be obtained by studying $Y_{\mu}$ for $\mu>\mu_{b}$.

Before give the initial one parameter family, some notations and definitions are given. For a curve $\gamma$, which lies in the plane $y=0$, it is defined the set $\Sigma(\gamma)=\{(x, y, z):(x, 0, z) \in \gamma,|y| \leq 1\}$. Given any small, positive fixed numbers $\delta<\delta^{\prime}$ we consider two functions $C^{+}, C^{-}:\left[-\delta,-\delta^{\prime}\right] \rightarrow R^{+}, R^{-}$with the following properties: $C^{ \pm}\left(-\delta^{\prime}\right)= \pm 1, C^{ \pm}(-\delta)= \pm e$ with $e<1$ close to 1 . In addition, $\left(C^{ \pm}\right)^{\prime}\left(-\delta^{\prime}\right)=0,\left(C^{+}\right)^{\prime}(-\delta)=-\infty,\left(C^{-}\right)^{\prime}(-\delta)=\infty,\left(C^{+}\right)^{\prime}(z) \leq 0$ and $\left(C^{-}\right)^{\prime}(z) \geq 0$. Let us define the curve $\gamma_{0}$ as the following one

$\{1\} \times\{0\} \times\left[-\delta^{\prime}, \Delta_{z}\right] \cup C_{+} \cup[-e, e] \times\{0\} \times\{-\delta\} \cup C_{-} \cup\{-1\} \times\{0\} \times\left[-\delta^{\prime}, \Delta_{z}\right]$

where $C_{ \pm}=\left\{(x, 0, z):(x, 0, z) \in \gamma_{0}\right\}$, and $\Delta_{z}$ is a positive fixed number. Let us consider the set $\Sigma_{0}=\Sigma\left(\gamma_{0}\right)$ which is showed in the picture below

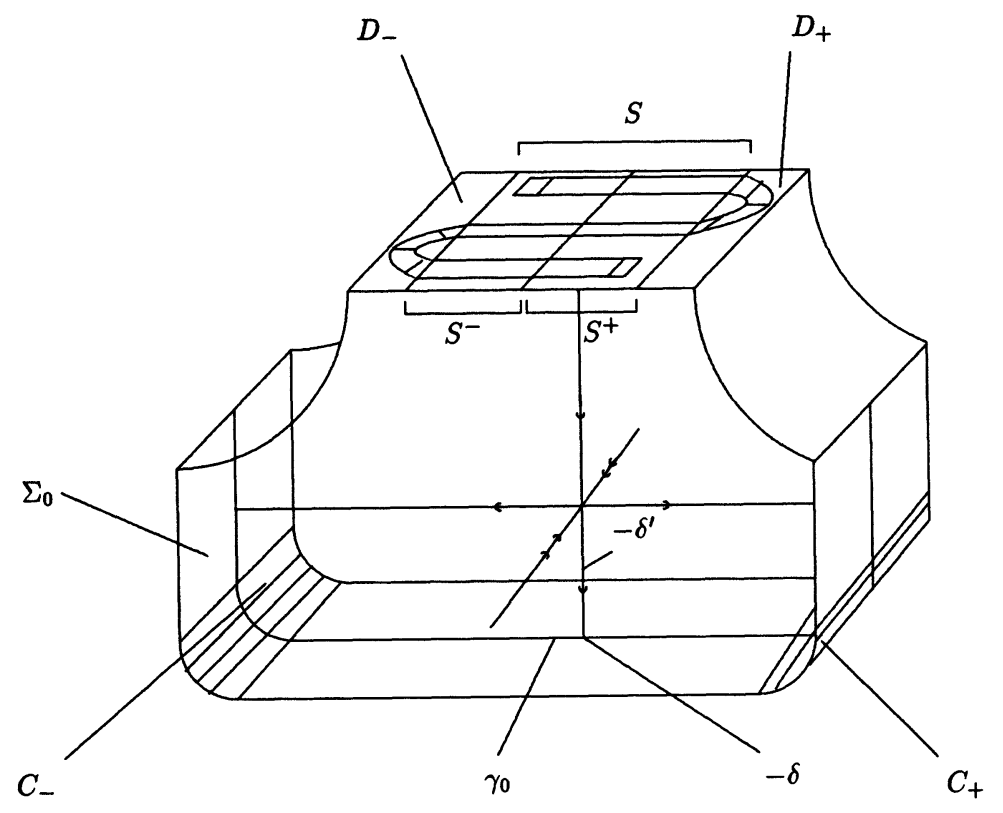

Fig. 3.

Here, the sets $C_{ \pm}$represent the "corners" of $\Sigma_{0}$. Let $S$ and $S^{*}$ be as these in H-1 $\S 2$, and let $D_{+}$and $D_{-}$be two small rectangles abyacent to 
the sides $\left\{x_{0}= \pm 1 / 2\right\}$ (see the picture above). Now, our initial vector field $X_{0}$ is defined in such a way it satisfies the following properties:

C-1) The hypotheses $\mathrm{H}-1, \mathrm{H}-2$ of $\S 2$ hold for $X_{0}$.

C-2) The map $\pi_{0}$ (see $\left.\mathrm{H}-2\right)$ is also defined on $D_{ \pm}$. It has the same form as the one given in $\mathrm{H}-2$ and $\pi_{0}\left(D_{ \pm}\right) \subset \operatorname{int}(S)$. See the picture below.

C-3) A successive flow-defined map, which is still denoted by $\pi_{f a r, 0}$, is defined from $\Sigma_{0} \backslash\{z \geq 0\}$ to $\tilde{S}=D_{ \pm} \cup S$. It sends the corners $C_{ \pm}$ into the interior of $D_{ \pm}$and it also carries the lines $x=\mathrm{cnt}$. and $z=\mathrm{cnt}$. in $\Sigma_{0}^{ \pm}=\Sigma_{0} \backslash\left(\{z \geq 0\} \cup C_{ \pm}\right)$into lines $x_{0}=$ cnt. in $\tilde{S}$, in a specific contractive way. In addition, the curves $y=\mathrm{cnt}$. in $\Sigma_{0}^{ \pm}$are carried into lines $y_{0}=$ cnt. in $\tilde{S}$. It is assumed that the image of the curves $y=\mathrm{cnt}$. in the whole $\Sigma_{0}$ have quadratic contact with the vertical foliation $\left\{x_{0}=\right.$ cnt. $\}$ in $\tilde{S}$. See the picture below

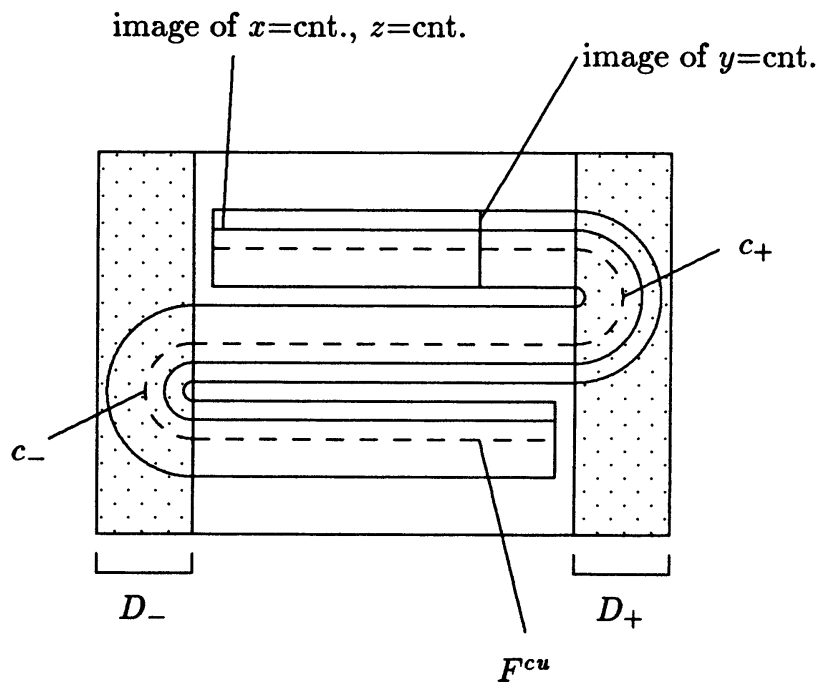

Fig. 4.

Here, $F^{c u}$ represents the intersection of $W^{u}(0,0,0)$ with $\tilde{S}$. The hypotheses above imply that this set is a nice curve in the sense of it is horizontal in $\tilde{S}$, although the whole curve $F^{c u}$ has two critical points denoted by $c_{+}, c_{-}$with respect to the vertical foliation in $\tilde{S}$. These critical points are generic (i.e. $c_{ \pm}$have quadratic contact with the above mentioned foliation).

As before, we set $X_{0}$ into the family $X=\left\{X_{\mu}\right\}_{\mu \in[-1,1]}$ which takes the form (1) in the neighborhood of $[-1,1]^{3}$ which comes from $\S 2$. We try to study the perturbations of $X$ in the space of one-parameters families as 
was done before. In the sequel, we suppose that $X_{0}$ is generic (in the sense of the Theorem 2.2). We denote by $F_{\mu}^{c u}, c_{-, \mu}, c_{+, \mu}$, etc. the analytical continuation of the corresponding elements of $X_{0}$ above descrited. We do not use notations related to perturbation arcs $Y=\left\{Y_{\mu}\right\}_{\mu \in[-1,1]}$ close to X.

Let us give the following Lemma.

LEMMA 3.1. - Let $U$ be a small neigbourhood of $X$ in the space of oneparameter families of vector fields. Then there exists a residual set $U$ ' in $U$ for which the following hold:

If $Y$ is a close family as before in $U^{\prime}$ and $\mu_{b} \in(-1,1)$ is as that in the Proposition 2.7, then the positive orbit of $c_{+, \mu}$ and $c_{-, \mu}$ under the map $\pi_{0}$ has empty intersection with $W^{s}\left(o_{\mu_{b}}\right)$; where $o_{\mu_{b}}$ is the analytical continuation of the saddle-node $(0,0,0)$ of $X_{0}$.

Remarks.

1) In other words, the Lemma 3.1 says that for a residual set $U$ ' in $U$ there is no critically twisted orbit, that is, there is no tangency orbits between $W_{\mu_{b}}^{s}\left(o_{\mu_{b}}\right)$ and $W_{\mu_{b}}^{c u}\left(o_{\mu_{b}}\right)$. See for instance [7] where critically twisted orbit is studied but associated to hyperbolic saddle type singularities.

2) For every arc $Y=\left\{Y_{\mu}\right\}_{\mu \in[-1,1]}$ close to $X$, it is defined a first return map $\pi_{\mu}$ associated to $Y_{\mu}$. It is a discontinuous map, defined in $\tilde{S} \backslash\left\{x_{0}=0\right\}$, if $\mu \leq 0$ (provided $\mu_{b}=0$ for every near arc $Y$ ) and $\pi_{0}$ is as the one given in $\mathrm{C}-2$. When $\mu>0$, these maps become diffeomorfisms all of them defined in the whole set $\tilde{S}$. The main purpose here is to describe this set of maps. These have the form $\pi_{\mu}=\pi_{f a r, \mu} \circ \pi_{l o c, \mu}$ where $\pi_{l o c, \mu}$ is the local map from $\tilde{S}$ to $\Sigma_{0}$ and $\pi_{f a r, \mu}$ is the global map from $\Sigma_{0}$ to $\tilde{S}$. The first one has the form

$$
\pi_{l o c, \mu}\left(x_{0}, y_{0}\right)=\left(x\left(\tau_{0}, x_{0}, \mu\right), y\left(\tau_{0}, y_{0}, \mu\right), z\left(\tau_{0}, \mu\right)\right)
$$

where again $\tau_{0}$ is defined by the implicit equation

$$
\left(x\left(t, x_{0}, \mu\right), y\left(t, y_{0}, \mu\right), z(t, \mu)\right) \in \Sigma_{0}
$$

and the solution $(x, y, z)$ comes from $\S 2$. A detailed description of this map will be given in the next section.

To continue we will need the following definitions

Definition. - A vertical band or simply a band is a set in $\tilde{S}$ given by

$$
B=\left\{\left(x_{0}, y_{0}\right) \quad \ni \quad y_{0} \in[-1-d, 1+d] ; \varphi_{1}\left(y_{0}\right) \leq x_{0} \leq \varphi_{2}\left(y_{0}\right)\right\}
$$

where $\varphi_{i}$ are almost vertical $C^{r}$-functions (i.e. with small derivative) with $\varphi_{1}<\varphi_{2}$. We call these functions the boundary of B. When $\varphi_{i}<0(\mathrm{i}=1,2)$ 
we say that $\mathrm{B}$ is a negative band. In the positive case we say that it is a positive band. If $\mathrm{B}$ is a band, the number $\left|\varphi_{1}-\varphi_{2}\right|$ is called the diameter of $B(|$.$| is the supremum norm).$

The next proposition is key in what follows. Recall that we are working on with perturbations as that in (2) (see §2).

Proposition 3.2. - For arcs $Y$ near $X$ and for $\mu>0$ small, there exists three bands $B_{\mu}, B_{\mu}^{l}$ and $B_{\mu}^{r}$ with $B_{\mu} \supseteq\left(B_{\mu}^{l} \cup B_{\mu}^{r}\right)$ and $B_{\mu}^{l(r)}$ negative (positive) band such that the following statements hold:

a) There exist a $\pi_{\mu}$-invariant contractive almost vertical foliation $\mathcal{F}_{\mu}^{s}$ defined in $H_{\mu}=B_{\mu} \backslash\left(\operatorname{int}\left(B_{\mu}^{l}\right) \cup \operatorname{int}\left(B_{\mu}^{r}\right)\right)$, where $\pi_{\mu}$ is the continuation of $\pi_{0}$ for the perturbation arc $Y$ (see remark after the Lemma 3.1).

b) The maximal invariant set in $H_{\mu}$ given by

$$
\triangle_{\mu}=\cap_{n \in Z} \pi_{\mu}^{n}\left(H_{\mu}\right)
$$

is a nontrivial saddle type basic set of $\pi_{\mu}$.

c) The diameters of $B_{\mu}^{l(r)}$, and that of the convex hull of the union $B_{\mu}^{l} \cup B_{\mu}^{l}$ are of order $K e^{-c / \sqrt{\mu}}$ for some positive fixed constants $K$ and $c$.

We give the proof of the Proposition 3.2 through the following lemmas.

LemmA 3.3. - For perturbations arcs $\left\{Y_{\mu}\right\}_{\mu \in[-1,1]}$ as that in (2) $\S 2$ and for $\mu>0$ there exists a band $B_{\mu}$ which is fenced by stable manifolds of periodic orbits of $\pi_{\mu}\left(B_{\mu} \subset \tilde{S}\right)$ such that

a) There exist two band $B_{\mu}^{l}$ and $B_{\mu}^{r}$ (in $S$ ) negative and positive (resp.) so that $B_{\mu} \cap \pi_{\mu}^{-1}\left(B_{\mu}\right)=B_{\mu} \backslash\left(\operatorname{int}\left(B_{\mu}^{r}\right) \cup \operatorname{int}\left(B_{\mu}^{l}\right)\right)$

b) The boundary of both bands are part of the stable manifolds which fence $B_{\mu}$

c) The diameters of $B_{\mu}^{l(r)}$ and of the convex hull of $B_{\mu}^{l} \cup B_{\mu}^{r}$ are of the order $K e^{-c / \sqrt{\mu}}$ for some positive constants $K$ and c.

Proof. - Take the band $B_{\mu}$ (for $\mu \geq 0$ ) as follows (see fig. 5)

Here the boundary of $B_{\mu}$ is union of two parts of stable manifolds of periodic points of $\pi_{\mu}$. Now, for $\mu=0$ define the corners $\mathcal{C}_{1}$ and $\mathcal{C}_{2}$ as $\mathcal{C}_{1} \cup \mathcal{C}_{2}=B_{0} \backslash W^{c u}(\theta) \cap\left(D_{-} \cup D_{+}\right)$, where $\theta$ is the analytical continuation of the saddle-node $(0,0,0)$ of $X_{0}$.

We would like to see $B_{\mu} \backslash \pi_{\mu}^{-1}\left(B_{\mu}\right)$, for $\mu>0$. Just looking at the normal form (2) and using the relation (4) to compute $\pi_{l o c, \mu}$ we can observe that, the set of points in $B_{\mu}$ which are carried by the local map into the union of $\mathcal{C}_{1}$ and $\mathcal{C}_{2}$ are just two bands (nearly vertical ones). Now, it can be proved that these two bands are contained into two bands $B_{\mu}^{l(r)}$ which are fenced by the same stable manifold of the boundary of $B_{\mu}$. 


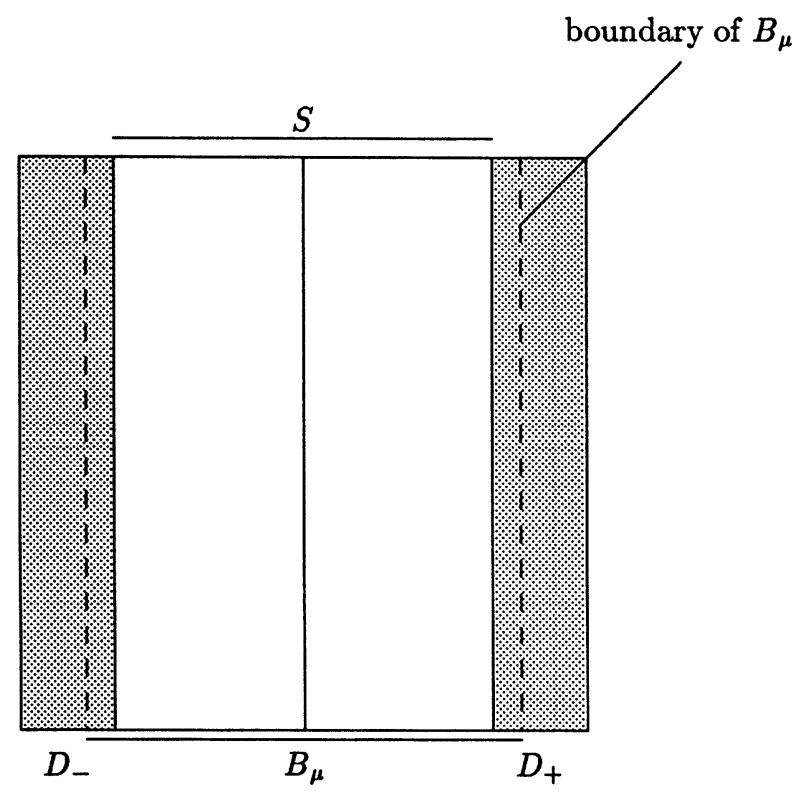

Fig. 5.

The map $\pi_{\mu}$ restricted to $B_{\mu}$ looks like a horseshoe map. Indeed, we have the picture $(\mathrm{g})$ below for $\pi_{\mu}$

The problem here is related with the image of $\mathrm{L}$ (in the picture, it is denoted by L') which does not cross the whole set $\mathrm{L}$ (but it cross $\mathrm{N}$ and M). The same observation holds for N. Finally M cross L,N and M itself. Recall that the boundary of all these bands are part of stable manifold of periodics orbits of $\pi_{\mu}$.

LemMa 3.4. - For $\mu>0$ small there exists a $\pi_{\mu}$-invariant nearly vertical contractive foliation $\mathcal{F}_{\mu}^{s}$ defined in $B_{\mu} \backslash\left(\operatorname{int}\left(B_{\mu}^{l}\right) \cup \operatorname{int}\left(B_{\mu}^{l}\right)\right)$. The boundaries of these bands are part of $\mathcal{F}_{\mu}^{s}$. The one dimensional foliation map induced by $\pi_{\mu}$ has the form

$$
f:[0, a] \cup[b, c] \cup[d, 1] \longrightarrow[0,1]
$$

with $f(0)>0 ; f(1)<1 ; f(a)=f(b)=1 ; f(c)=f(d)=0$. The function $f$ is a differentiable map and $\left|f^{\prime}\right| \geq \lambda>1$ for some constant $\lambda$. If we define $\triangle_{f}$ as

$$
\triangle_{f}=\cap_{n=0}^{\infty} f^{-n}([0,1])
$$

then it is a Cantor set, $f$ restricted to $\triangle_{f}$ is a topologically transitive map and $C L(\operatorname{Per}(f)) \supseteq \triangle_{f}$. Moreover, $\triangle_{f}$ is an hyperbolic set for $f$ and 0 and 1 are preperiodics points of $f$ (see picture $(h)$ below). 


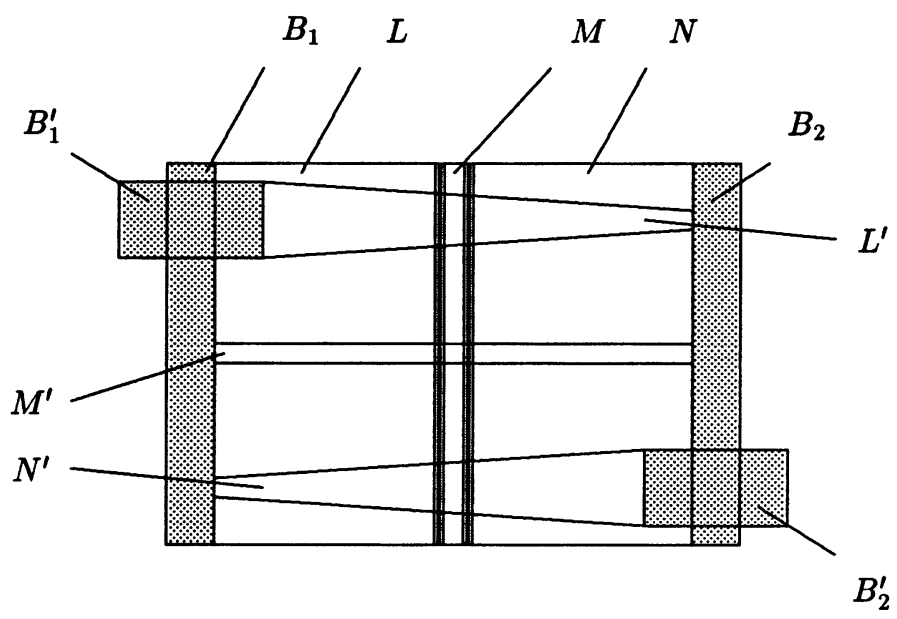

(g)

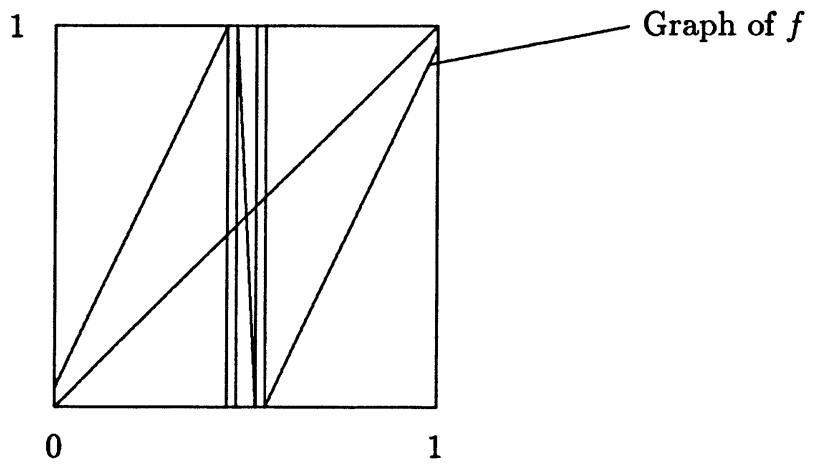

(h)

Fig. 6.

Proof. - First, let us explain how the proof works. We apply the arguments given in [2] and [15] to a appropriate extension $\pi_{\mu}^{e x t}$ of $\pi_{\mu}$, in order to obtain a $C^{1}, \pi_{\mu}^{e x t}$-invariant foliation $\mathcal{F}_{\mu}^{e x t}$ defined in a band $B_{\mu}^{e x t}$ which will contain $B_{\mu}$. The boundaries of $B_{\mu}, B_{\mu}^{l}$ and $B_{\mu}^{r}$ will be leaves of $\mathcal{F}_{\mu}^{e x t}$. Finally, the above-mentioned foliation restricted to $B_{\mu}$ will define a foliation for which the statement of the Lemma 3.4 holds. To start with the proof, one takes a band $B_{\mu}^{e x t}$ which is formed by adding two small bands $B_{1}$ and $B_{2}$ to $B_{\mu}$. Now, we define the map $\pi_{\mu}^{e x t}$ as being equal $\pi_{\mu}$ on $B_{\mu}$. Moreover, the image sets $B_{i}{ }^{\prime}$ of $B_{i}(i=1,2)$ under $\pi_{\mu}^{e x t}$ are as in the picture $(\mathrm{g})$ above. One can choose this extension map in such 
a way its derivative has the same hyperbolic properties attached to the corresponding derivative $D \pi_{\mu}$.

To continue, we fixed a $C^{1}$-foliation $R_{\mu}$ on suitable bands $B_{1}^{e x t}, B_{2}^{e x t}$, $B_{3}^{e x t}$ and $B_{4}^{e x t}$ which have the following properties:

1) $B_{1}^{e x t}$ is contained in $B_{1}$ ( $B_{2}^{e x t}$ is contained in $\left.B_{2}\right)$, the left (right) boundary of $B_{\mu}^{e x t}$ is contained in the boundary of $B_{1}^{e x t}\left(B_{2}^{e x t}\right)$.

2) $B_{3}^{\text {ext }}$ and $B_{4}^{\text {ext }}$ are contained in the interior of $B_{\mu}^{l}$ and $B_{\mu}^{r}$ respect.

Now, there exists a finite set of differentiable functions $\theta:[-1,1] \rightarrow R$ such that the union of the boundaries of $B_{i}^{e x t}(i=1, . ., 4)$ is equal to the union of the graph of the functions lying in the above-mentioned set of differentiable functions. Define $\mathcal{B}$ as the set of continuous maps $\varphi: B_{\mu}^{e x t} \rightarrow[-1,1]$ such that

1) $\varphi(q)=R_{\mu}(q)$ if $q$ lies in the union of the bands $B_{i}^{e x t}(i=1, . ., 4)$.

2) $\varphi(\theta(y), y)=\theta^{\prime}(y)$ for all $y$ and $\theta$.

It follows that every $\varphi \in \mathcal{B}$ define a vector field on $B_{\mu}^{\text {ext }}$ by setting

$$
\eta^{\varphi}(q)=(\varphi(q), 1)
$$

Let us write the expression of the $\pi_{\mu}^{e x t}$-induced graph transformed operator defined on $\mathcal{B}$. It is the following

$$
T(\varphi)=\left\{\begin{array}{l}
\frac{D_{\mu}^{e x t} \cdot\left(\varphi \circ \pi_{\mu}^{e x t}\right)-B_{\mu}^{e x t}}{A_{\mu}^{e x t}-C_{\mu}^{e x t} \cdot\left(\varphi \circ \pi_{\mu}^{e x t}\right)} \quad \text { on } B_{\mu}^{e x t} \backslash \cup_{i=1}^{4} B_{i}^{e x t} \\
R_{\mu} \quad \text { otherwise }
\end{array}\right.
$$

where the functions $A_{\mu}^{e x t}, B_{\mu}^{e x t}$,...etc. are the entries of $D \pi_{\mu}^{e x t}$ as these of $D \pi_{\mu}$ (see $\S 2$ ). It follows that a fixed point $\varphi_{0}$ of $T$ induce a continuous $\pi_{\mu}$ invariant foliation by integrating the corresponding vector field $\eta^{\varphi_{0}}$. Now, we endow $\mathcal{B}$ with the supremum norm. The main told for the existence of such a fixed point is that $T$ is a well defined contracting operator on $\mathcal{B}$. It is well defined because of one can prove the continuity of $T(\varphi)$ and moreover, $T(\mathcal{B}) \subset \mathcal{B}$ and $T$ is a contraction because of computations which are similar to these given in $\S 2$. These computations are based on hyperbolic properties of the entries $A_{\mu}^{e x t}, B_{\mu}^{e x t}$....etc. which are still valid when $\mu>0$ (see also the next section). To prove that $\varphi_{0}$ is a $C^{1}$-map we can still proceed as in the proof of the Proposition 2.3. Indeed, the operator $S(\varphi)(\mathcal{A})$ is defined as that given in $\S 2$, except it is equal to $\nabla R_{\mu}$ in $B_{i}^{e x t}(i=1, \ldots, 4)$.

Now, it can be proved that the foliation map, induced by $\pi_{\mu}$, is in fact a expansive map because the function $\left|A_{\mu}^{\text {ext }}\right|$ is large. Hence the hyperbolicity of $\triangle_{f}$ follows. The transitivity and the density of the periodics orbits is obtained through the next facts

a) $\triangle_{f}=[0,1] \backslash \cup_{n=0}^{\infty} I_{n}$, where $I_{n}=f^{-1}\left(I_{n-1}\right) \quad, I_{0}=(a, b) \cup(c, d)$ 
b) Every $I_{n}$ has the form $I_{n}=\cup_{i=1}^{r_{n}} I_{n}^{i}$, where the $I_{n}^{i}$,s is a closed interval and for every $i \in\left\{1, \ldots, r_{n}\right\}$ there exists a interval $J \subset I_{n}^{i}$ such that $f^{l}(J)=[0,1]$ for some positive integer $l$. Observe that the collection of $\left\{I_{n}^{i}\right\}$ is like a Markov partition (see [14]) of $\triangle_{f}$.

COROLlary 3.5. - The maximal invariant set given by

$$
H_{\mu}=\cap_{n \in Z} \pi_{\mu}^{n}\left(B_{\mu} \backslash\left(\operatorname{int}\left(B_{\mu}^{l}\right) \cup \operatorname{int}\left(B_{\mu}^{r}\right)\right)\right.
$$

is a hyperbolic basic set for $\pi_{\mu}, \mu>0$ small.

Now the Proposition 3.2 follows by using the Lemma 3.3, Lemma 3.4 and the Corollary 3.5 .

Let us start with the proof of the Theorem I. First, we introduce some notations. Take a function $\mathrm{f}$ as in Lemma 3.4 and set $D_{f}=$ "domain of $f$ " = $[0,1] \backslash I_{0}$ (recall the proof of the Lemma 3.4). We set index everywhere i.e. $f_{\mu}, I_{0, \mu}$ and so on.

There are also defined the critical points $c_{ \pm}$associated to the parameter $\mu=0$ (see remark before the Lemma 3.1). Set $c_{1}=\pi_{0}\left(c_{+}\right)$and so we have the next picture

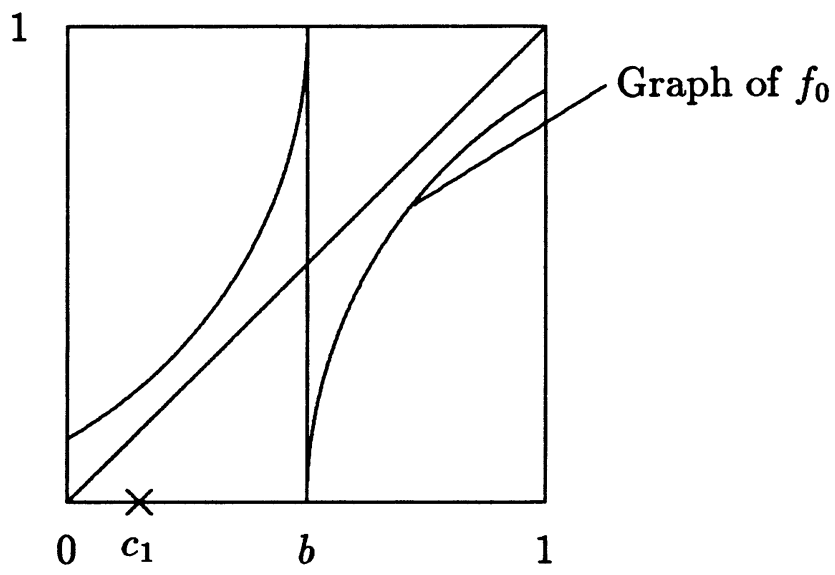

Fig. 7.

Here " $b$ " represents the lift which is part of the stable manifold (notice that $Y_{0}$ is a Saddle-node Lorenz vector field) of the saddle-node singularity $\theta$ given above (see the proof of the Lemma 3.3).

Remarks. - We want to point out the following: for all small $\mu>0$ there exists an almost horizontal tangency line $l_{\mu}$, which is close to $c_{1}$. It 
will contain a Cantor set $K_{\mu}$ formed by tangency points of $\mathcal{F}_{\mu}^{u}$ (the strong unstable foliation associated to the hyperbolic set $H_{\mu}$ given in Corollary 3.5) with the almost vertical foliation found in the Lemma 3.4. Also $l_{\mu} \rightarrow c_{1}$ for $\mu \rightarrow 0^{+}$in the Hausdorff topology. Let us denote $\left[e_{\mu}, h_{\mu}\right]$ the convex hull of $K_{\mu}$. Then $e_{\mu}$ and $h_{\mu}$ are accumulated by the unstable manifold of some fixed point of $\pi_{\mu}$ in $H_{\mu}$.

Back again to the proof of the Theorem I, take $Y=\left\{Y_{\mu}\right\}_{\mu \in[-1,1]}$ in the residual set given in Lemma 3.1 for a small neighborhood $U$ of the initial family X. We shall prove that $K_{\mu}$ and $\triangle_{\mu}=\triangle_{f_{\mu}}$ (see notation in Lemma 3.4) have a cross point for positive parameter values $\left(\mu_{k}\right) \rightarrow 0^{+}$, that is two curves $t(\mu) \in K_{\mu}$ and $l(\mu) \in \triangle_{\mu}$ such that $t(\mu)-l(\mu)$ has a unique zero in a small neighborhood of $\mu=\mu_{k}$ and $(t-l)^{\prime}\left(\mu_{k}\right) \neq 0$. It is clear that such a cross point give us a quadratic unfold in $\mu=\mu_{k}$.

The boundary points of every $I_{n, \mu}^{i}$ are points in $\triangle_{\mu}$ because 0 and 1 are preperiodics points of $f_{\mu}$. Also when $\mu \rightarrow 0^{+}$such a boundary points move on, with positive speed, to points which are preimage points of $b$ under the map $f_{0}$ (see the picture above). This implies that the intervals $I_{n, \mu}^{i}$ are closing on (when $\mu \rightarrow 0^{+}$). It can be proved that the speed of such a closing goes to zero when $\mu \rightarrow 0^{+}$but it is positive for $\mu>0$ (see Proposition 3.2 (c)). The same is true for the speed of convergence of $e_{\mu}$ and $h_{\mu}$ to $c_{1}$.

Let $\mu>0$ be small and look at $\left[e_{\mu}, h_{\mu}\right]$. If $e_{\mu^{*}}$ or $h_{\mu^{*}}$ are in the same gap for all $\mu^{*} \in(0, \mu)$ then $c_{1}$ meet $x_{0}=0$ for some $f_{0}$-iterate (this is a contradiction), thus $e_{\mu^{*}}$ has to change of gap. In this case we must have a cross point and so we are done. Suppose now that $e_{\mu}$ does not belong to any gap of $\triangle_{\mu}$, for parameters $\mu$ which accumulates $\mu=0$. In that case we must have a gap of $\triangle_{\mu}$ in the interval $\left(e_{\mu}, c_{1}\right)$. Using again that $e_{\mu} \rightarrow c_{1}$ and the $f_{0}$-orbit of $c_{1}$ does not meet $x_{0}=0$, we can show that there exists a cross point (in fact two of them). That finishes the proof of the Theorem I because now the Proposition 2.1 applies.

\section{PROOF OF THE THEOREM II}

In this section, a proof of the Theorem II will be given through the following short steps: First, a slightly modification (due to Newhouse [10]) of the maps given in [12] is considered. These maps, which will be called Plykin maps, naturally generate nontrivial axiom A attractors (see Lemma 10 below). Next, a initial one-parameter family of vector fields $X$ is constructed, in such a way $\mathrm{H}-1, \mathrm{H}-2$ of $\S 2$ together another additional 
hypotheses hold. Finally, it will be shown that for any arc $Y=\left\{Y_{\mu}\right\}_{\mu \in[-1,1]}$ close to $X$ and $\mu>0, Y_{\mu}$ generates a poincare map $\pi_{\mu}$ which will be indeed a Plykin map in its domain.

Let us consider a domain $\mathcal{D}$ as follows:

$$
F^{h}=\equiv \quad F^{v}=111,
$$

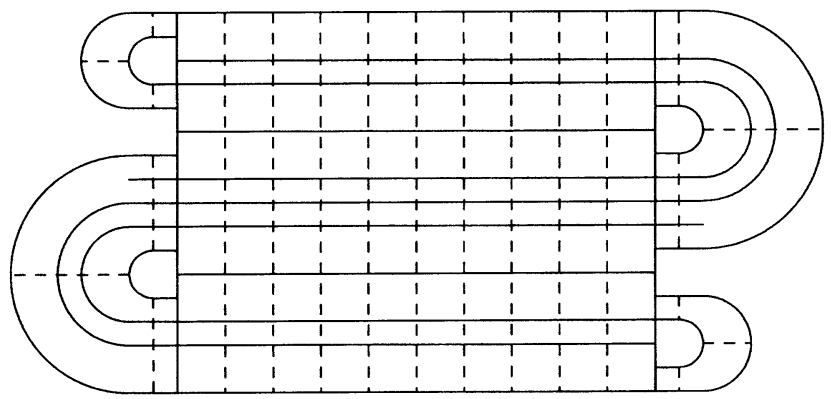

Fig. 8.

So it is about a plane disk with four holes. A vertical foliation $\left(\mathcal{F}^{v}\right)$ together with a horizontal foliation $\left(\mathcal{F}^{h}\right)$ are depicted above. Both foliations generate a coordinate system which we denoted by $\left(x_{0}, y_{0}\right)$ in the sequel.

We say that a diffeomorphism $F: \mathcal{D} \rightarrow \operatorname{Int}(\mathcal{D})$ is a Plykin Map iff it sends the set $\mathcal{D}$ into itself in the following geometrical way

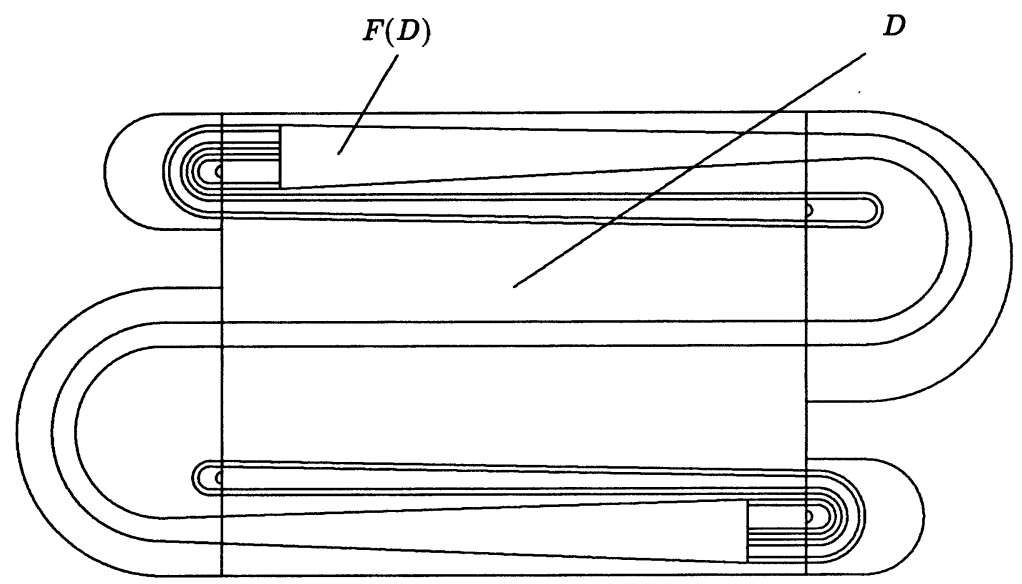

Fig. 9. 
Moreover, $F$ has the following form, with respect to the above-mentioned coordinate system $\left(x_{0}, y_{0}\right)$ :

$$
F\left(x_{0}, y_{0}\right)=\left(f\left(x_{0}, y_{0}\right), g\left(x_{0}, y_{0}\right)\right)
$$

with $\left|f_{x_{0}}\right|>\gamma ;\left|f_{y_{0}}\right|,\left|g_{y_{0}}\right|$ and $\left|g_{x_{0}}\right| \cdot\left|f_{x_{0}}\right|^{-1}<\lambda$ for some fixed positive constants $\lambda$ small and $\gamma>1$.

Now, using arguments which can be found in [2] and [12], we obtain the following Lemma.

Lemma 4.1. - Let $F$ be a Plykin map with domain $\mathcal{D}$. Then the set $\Delta_{F}$ defined by

$$
\Delta_{F}=\cap_{n \geq 0} F^{n}(\mathcal{D})
$$

is a nontrivial axiom A attractor for $F$.

Proof. - First, we shall prove the existence of a $C^{1}$-strong stable foliation. We proceed as before (see the last section). Consider the set of trial foliations $\{(\varphi, 1):|\varphi|<\epsilon\}$ and define the graph transformed operator as follows

$$
T(\varphi)=\frac{D \cdot(\varphi \circ F)-B}{A-C \cdot(\varphi \circ F)}
$$

where again $A, B, \ldots$ etc. are the entries of $D F$. Now the same computations as that in $\S 2$ shows that $|T(\varphi)|<\epsilon$ if $|\varphi|<\epsilon$ and $T$ is a contraction on the above set of trial foliations. As before, we can show that the corresponding fixed foliation, which exists because of the contracting map theorem, is actually a $C^{1}$-map. Next we use the criterium of Hirsch and Pugh [5] to prove that the foliation map is indeed a expanding map. Notice that the inequalities below hold because of $F$ is a Plykin map

$$
A>1 ; \frac{2|B|+|D|}{|A|}<\epsilon<1 ; \frac{|B|+|C|+|D|}{|A|}<\beta<1
$$

with $\epsilon, \beta$ being small constants. Then propositions 4.7 and 4.10 of [5] apply in order to obtain that $\Delta_{F}$ is in fact a hyperbolic set. Finally, we consider the branched manifold [20] generates by the invariant foliation found above. The quotient map generates by $F$ in such a branched manifold will be a expansive map so the set $\Delta_{F}$ is a nontrivial basic set (i.e. $F / \Delta_{F}$ is a transitive map). This finishes the proof of the Lemma 4.1.

Now, a initial vector field is defined. Once more, we use the scheme given in $\S 3$. Let us consider a domain $\mathcal{D}_{0}$ as that given in the pictures 
above but containing the square $[-1 / 2,1 / 2]^{2}$. In addition, we consider the three dimensional set $\mathcal{D}_{0} \times\{1\}$ which is still denoted by $\mathcal{D}_{0}$ and a vector field $X_{0}$ satisfying the following assumptions:

PL-1) The hypotheses $\mathrm{H}-1, \mathrm{H}-2$ of $\S 2$ hold for $X_{0}$.

PL-2) The map $\pi_{0}$ (see H-2) is also defined in the whole set $\mathcal{D}_{0}$. The image set $\pi_{0}\left(\mathcal{D}_{0}\right)$ is contained in $\mathcal{D}_{0}$ as follows

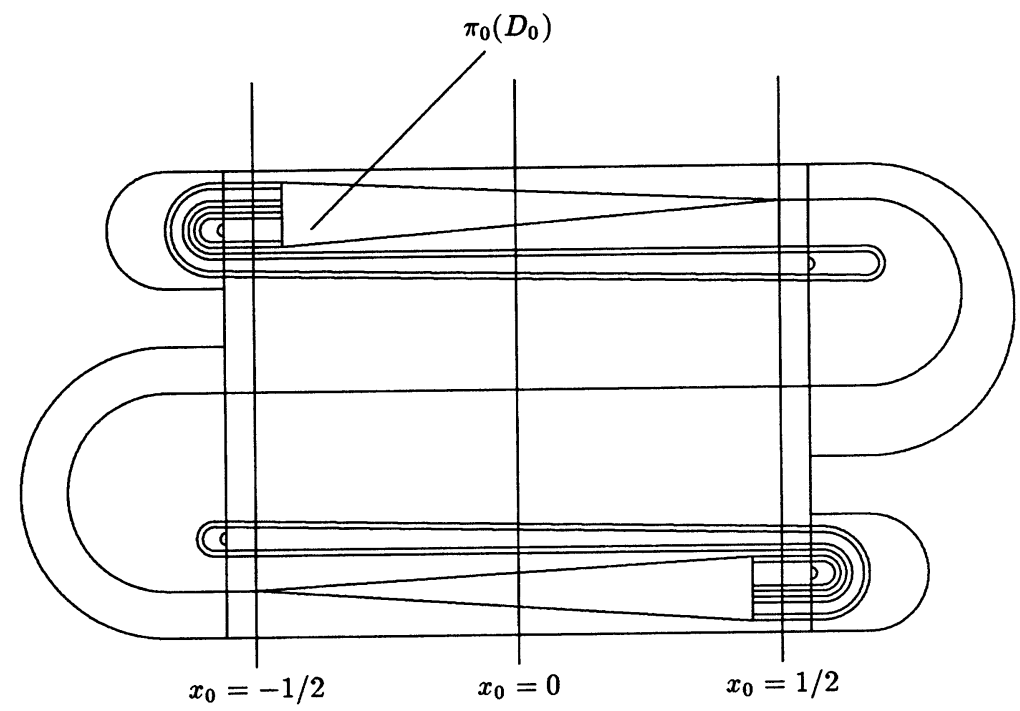

Fig. 10.

In addition, the map $\pi_{0}$ has the following form $\pi_{0}\left(x_{0}, y_{0}\right)=$ $\left(f_{0}\left(x_{0}\right), g_{0}\left(x_{0}, y_{0}\right)\right)$ with

a) The function $f_{0}$ is a Lorenz unidimensional map (i.e. satisfies (c) and (d) of $\mathrm{H}-2$ $\S 2$ ) when it is restricted to $[-1,1]$. Besides this property, it is a expanding map in the whole branched manifold induced by $\mathcal{F}^{v}$ (see [20]) which is showed below

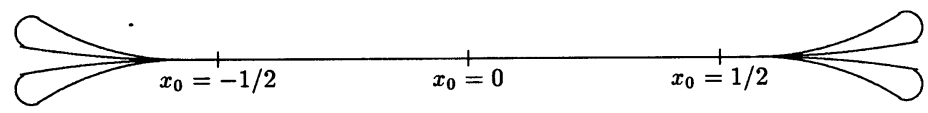

Fig. 11.

b) The following inequalities hold: $\left|f_{0, x_{0}}\right| \geq \gamma$ and $\left|f_{0, y_{0}}\right|,\left|g_{0, y_{0}}\right|,\left|g_{0, x_{0}}\right| .\left|f_{0, x_{0}}\right|^{-1}<\lambda$, where the constants $\lambda$ and $\gamma$ are as these in the definition of a Plykin map given above. 
PL-3) It is defined a flow-defined function $\pi_{f a r, 0}$ from a small fixed neighborhood of the set $\Sigma_{0} \backslash\{z \geq 0\}$ (see notation in $\S 3$ ) to $\mathcal{D}_{0}$. It carry the lines $x=\mathrm{cnt}$., $z=\mathrm{cnt}$. in this set into lines $x_{0}=\mathrm{cnt}$. in $\mathcal{D}_{0}$ in a specific contractive way. Moreover, the curves $y=\mathrm{cnt}$. in the above considered neighborhood are carried into lines $y_{0}=$ cnt. in $\mathcal{D}_{0}$.

To construct the open set in the statement of the Theorem II, we set $X_{0}$ in a one-parameter family $X=\left\{X_{\mu}\right\}_{\mu \in[-1,1]}$ as that in (1) $\S 2$. We suppose that $X_{0}$ is generic, in the sense of the Theorem $2.2 \S 2$ and so nearby arcs $Y$ are as these in (2) $\S 2$. As was pointed out before, every arc $Y$ generates a one-parameter family of maps $\left\{\pi_{\mu}\right\}_{\mu \in[-1,1]}$ and our goal here is to prove that $\pi_{\mu}$ is a Plykin map when $\mu>0$.

The Lemma below will be used to obtain estimates related to the derivative of the local map $\pi_{l o c, \mu}$ induced by $Y$. Recall the notations in the Theorem $2.2 \S 2$.

Lemma 4.2. - Suppose that $\alpha>0$ is arbitrary. Then there exists a constant $\delta_{0}>0$ such that if we define, for $\mu, \Delta>0$

$$
\rho_{\mu}(\Delta)=\inf _{z \in\left[-\delta_{0}, \Delta\right]}\left|H(z, \mu) e^{-\alpha \int_{z}^{1} \frac{d s}{H(s, \mu)}}\right|
$$

then, for any real number $M>0$ there exist $\mu_{0}, \Delta_{0}>0$ such that $\rho_{\mu}(\Delta)>M$ if $0<\mu<\mu_{0}$ and $0<\Delta<\Delta_{0}$.

Proof. - It can be proved that for every $\epsilon>0$ there exist $\delta_{0}>0$ and $v>0$ such that

$$
1-\epsilon \leq \frac{-H(z, \mu)}{\mu+a z^{2}} \leq 1+\epsilon
$$

if $(z, \mu) \in\left[-\delta_{0}, \delta_{0}\right] \times(0, v]$. Then Lemma 4.2 follows provided it holds with $\mu+a z^{2}$ instead of $-H(z, \mu)$ (see the arguments in [3]). Now

$$
\int_{z}^{1} \frac{d s}{\mu+a s^{2}}=(a \mu)^{-1 / 2}\{\operatorname{arctg}(\sqrt{a / \mu})-\operatorname{arctg}(\sqrt{a / \mu} \cdot z)\}
$$

then, it follows that

$$
\lim _{\mu \rightarrow 0^{+}} \sqrt{a \mu} \int_{z}^{1} \frac{d s}{\mu+a s^{2}}= \begin{cases}\pi / 2 & \text { if } z=0 \\ \pi & \text { if } z \in\left[-\delta_{0}, 0\right)\end{cases}
$$

therefore, all we need to check is the case $z \in(0, \Delta]$, where we choose $\Delta$ better than $\delta_{0}$. It must be proved that the quantity

$$
\inf _{z \in(0, \Delta]}\left\{\left(\mu+a z^{2}\right) \cdot e^{(a \mu)^{-1 / 2}[\operatorname{arctg}(\sqrt{a / \mu})-\operatorname{arctg}(\sqrt{a / \mu} \cdot z)]}\right\}
$$


is large. For this purpose, it is easy to prove that

$$
\operatorname{arctg}(\sqrt{a / \mu})-\operatorname{arctg}(\sqrt{a / \mu} . z)=\operatorname{arctg}\left(\frac{\sqrt{a \mu} \cdot(1-z)}{\mu+a z}\right)
$$

Suppose that $0<z \leq \sqrt{\mu / a}$. Then

$$
\left(\mu+a z^{2}\right) e^{(a \mu)^{-1 / 2}[\operatorname{arctg}(\sqrt{a / \mu})-\operatorname{arctg}(\sqrt{a / \mu} \cdot z)]} \geq e^{(\pi / 2-\epsilon-\operatorname{arctg}(1))(a \mu)^{-1 / 2}} \cdot \mu
$$

for some small positive number $\epsilon$. Choose $v_{1}>0$ such that $e^{R / \sqrt{a \mu}} \cdot \mu>M$ for $0<v<v_{1}$, with $R=\pi / 2-\epsilon-\operatorname{arctg}(1)>0$ and we are done. Now, suppose that $\Delta \geq z>\sqrt{\mu / a}$. Then $0<\frac{\sqrt{a \mu} .(1-z)}{\mu+a z} \leq 1$. On the other hand

$$
\operatorname{arctg}\left(\frac{\sqrt{a \mu} \cdot(1-z)}{\mu+a z}\right)=\cos ^{2}(\operatorname{arctg}(\xi))\left(\frac{\sqrt{a \mu} \cdot(1-z)}{\mu+a z}\right)
$$

for some $\xi \in[0,1]$. Therefore $\cos ^{2}(\operatorname{arctg}(\xi))$ is bounded away from zero and so

$$
\left(\mu+a z^{2}\right) e^{(a \mu)^{-1 / 2}[\operatorname{arctg}(\sqrt{a / \mu})-\operatorname{arctg}(\sqrt{a / \mu} \cdot z)]} \geq e^{\frac{K}{\mu+a z}} \cdot\left(\mu+a z^{2}\right)
$$

for some fixed positive constant $K$. But

$$
e^{\frac{K}{\mu+a z}} \cdot\left(\mu+a z^{2}\right)>a z^{2} e^{\frac{K}{a z^{2}+a z}}
$$

and this last expression is large, if $\Delta$ is small.

Corollary 4.3. - There exist $M>0$ and $\mu_{0}>0$ such that

$$
\left|\frac{\dot{y}\left(t, y_{0}, \mu\right)}{\dot{z}(t, \mu)}\right| \leq M
$$

if $0<t \leq \tau_{0}$ and $\mu \in\left(0, \mu_{0}\right]$.

The next Lemma deals with the one-parameter family of poincare maps $\pi_{\mu}^{*}:\left\{\left|x_{0}\right|<\Delta\right\} \rightarrow R$ given by $\pi_{\mu}^{*}\left(x_{0}\right)=\left(x\left(\tau_{0}, x_{0}, \mu\right), z\left(\tau_{0}, \mu\right)\right)$ where the functions $x\left(\tau_{0}, x_{0}, \mu\right), z\left(\tau_{0}, \mu\right)$ are respectively the first and the last component of $\pi_{\mu}$. Observe that $\tau_{0}$ is defined as the solution of $x\left(t, x_{0}, \mu\right) \in \gamma_{0}$. Here, $\gamma_{0}$ comes from the definition of $\Sigma_{0}$ (see beginning of $\S 3)$. With this in mind, there are defined two positive numbers $s_{\mu}$ and $t_{\mu}\left(s_{\mu}>t_{\mu}\right)$ such that $\pi_{\mu}^{*}\left(s_{\mu}\right)=\left(1,-\delta^{\prime}\right)$ and $\pi_{\mu}^{*}\left(t_{\mu}\right)=(e,-\delta)$.

LEMma 4.4. - There exist two small positive number $\delta, \delta^{\prime}\left(\delta>\delta^{\prime}\right)$ such that the following hold: there exists a positive number $M$ such that

$$
\frac{\left\|\left(\pi_{\mu}^{*}\right)^{\prime}\left(x_{0}\right)\right\|}{\left|x_{x_{0}}\left(\tau_{0}, x_{0}, \mu\right)\right|} \geq M
$$


for all small $\mu>0$ and $x_{0} \in\left[t_{\mu}, s_{\mu}\right]$.

Proof. - It is easy to see that (denoting $z=z\left(\tau_{0}, \mu\right)$, etc)

$$
\left(\pi_{\mu}^{*}\right)^{\prime}\left(x_{0}\right)=H(z, \mu) \tau_{x_{0}}\left(\left(C^{+}\right)^{\prime}(z), 1\right)
$$

where $C^{+}$comes from the definition of $\gamma_{0}$. Therefore, it follows that

$$
\left\|\left(\pi_{\mu}^{*}\right)^{\prime}\left(x_{0}\right)\right\|=\left|x_{x_{0}}\right| \cdot \frac{\sqrt{1+\left(C^{+}\right)^{\prime 2}(z)}}{\left|\left(C^{+}\right)^{\prime}(z)-\left[\left(\lambda_{1}+f(z, \mu)\right) / H(z, \mu)\right] . x\right|}
$$

Finally, we choose $M>0$ such that $\sqrt{1+\xi^{2}} /(\xi+B)>M$ for all $\xi>0$, where $B=\sup _{z \in\left[-\delta,-\delta^{\prime}\right]}\left|\frac{\lambda+f(z, \mu)}{H(z, \mu)}\right|$ (and so it does not depend on $\mu, z$ ). This concludes the proof of the Lemma 4.4

Proof of the Theorem II. - As was pointed out before, we must prove that $\pi_{\mu}$ is a Plykin map for small parameter $\mu>0$. A straightforward computation shows that

$$
D \pi_{\mu}=\left(\begin{array}{ll}
\tilde{b}_{\mu} \dot{y} \tau_{x_{0}}+<\left(\tilde{a}_{\mu}, \tilde{c}_{\mu}\right),\left(\pi_{\mu}^{*}\right)^{\prime}\left(x_{0}\right)> & \tilde{b}_{\mu} y_{y_{0}} \\
\tilde{e}_{\mu} \dot{y} \tau_{x_{0}}+<\left(\tilde{d}_{\mu}, \tilde{f}_{\mu}\right),\left(\pi_{\mu}^{*}\right)^{\prime}\left(x_{0}\right)> & \tilde{e}_{\mu} y_{y_{0}}
\end{array}\right)
$$

where again $\tilde{a}_{\mu}, \tilde{b}_{\mu}, \ldots$ etc. are the entries of $\pi_{f a r, \mu}$ and $\langle\cdot, \cdot\rangle$ denotes the scalar product in $R^{2}$. Here, $\pi_{l o c, \mu}$ is the continuation of the map $\pi_{l o c, 0}$ showed before (see PL-3). It follows from PL-3 that

a) $<\left(\tilde{d}_{0}, \tilde{f}_{0}\right), U>=0$

b) $\left|<\left(\tilde{a}_{0}, \tilde{c}_{0}\right), U>\right|$ is bounded away from zero

c) $\left|\tilde{e}_{0}\right|$ is small

d) $\tilde{b}_{0}=0$

for every unitary vector $U$ which belongs to the $\gamma_{0}$ 's direction. Now, using Lemma 4.2 and the Corollary 4.3 above we obtain $\left|\dot{y} \tau_{x_{0}}\right| /\left\|\left(\pi_{\mu}^{*}\right)^{\prime}\left(x_{0}\right)\right\|$ is a bounded function. Moreover, $\left\|\left(\pi_{\mu}^{*}\right)^{\prime}\left(x_{0}\right)\right\|$ goes to infinity, if $x_{0}$ goes to zero because of the Lemma 4.4. In addition, $\left|\tilde{b}_{\mu} y_{y_{0}}\right|$ and $\left|\tilde{e}_{\mu} y_{y_{0}}\right|$ are bounded, with small bounds. This finishes the proof of the Theorem II because of Lemma 4.1 applies.

Very recently a separation (i.e. there exists a codimension one separating lamina, see $\$ 1$ ) between the set of Morse-Smale flows and those Axiom A fields exhibiting a solenoid-like attractor (see [16]) was proved to exist by Shilnikov and Turaev, in the space of flows of any $n$-dimensional manifold with $n \geq 4$. The methods given in this research can be applied in order to prove the following statement: There exists a separation between the set of fields displaying a multidimensional Lorenz attractor and the set of Axiom A flows with an attractor of solenoid type, in the flow's space of any manifold of dimension greather or equal than four. 


\section{ACKNOWLEDGEMENTS}

The author acknowledges fruitful conversations with Jacob Palis Jr., Alvaro Rovella, Enrique Pujals, Bernardo San Martin and Ismael Huerta. I would like to thank Ismael Huerta by his helpfull computer assistance.

\section{REFERENCES}

[1] V. S. Afraimovic and L. P. Shilnikov, On Attainable Transitions from Morse-Smale systems to systems with many periodic motions, Math. U.S.S.R. Izv., Vol. 8, 1974, N. 6, pp. 1235-1270.

[2] R. Bamon, R. Labarca, R. Mañe and M. J. Pacifico, The explosion of Singular Cycles, Publ. Math. I.H.E.S., Vol. 78, 1993, pp. 207-232.

[3] L. DiaZ, J. Rocha and M. Viana, Saddle Node cycles and prevalence of Strange Attractors, Preprint I.M.P.A. to appear.

[4] J. Guckemheimer and R. F. Williams, Structural Stability of Lorenz Attractor, Pub. Math. I.H.E.S., Vol. 50, 1979, pp. 59-72.

[5] M. Hirsch and C. Pugh, Stable Manifold and Hyperbolic sets, Global Analysis, Proc. Sym. Pure Math., Vol. 14.

[6] M. Hirsch, C. C. Pugh and M. Shub, Invariant Manifold, Lec. Not. in Math., Vol. 583, 1977.

[7] M. KisaKa, H. KoKubu and H. OKa, Bifurcations to N-homoclinic orbits and N-periodic orbits in vector field, Journal of Dynamics and Differential Equations, Vol. 5 (2), 1993.

[8] E. N. LorenZ, Deterministic non-periodic flow, J. Atmos. Sci., Vol. 20, 1963, pp. 130-141.

[9] L. Mora and M. Viana, Abundance of Strange Attractors, Act. Math., Vol. 171, 1993, pp. $1-71$.

[10] S. Newhouse, Lectures on dynamical systems, Progress in Math, N. 8, Birkhauser-Boston. Boston.

[11] S. Newhouse, D. Ruelle and F. Takens, Occurrence of Strange Axiom A Attractors Near Quasi Periodic Flows on $T^{m}, m \geq 3$, Commun. math. Phys., Vol. 64, 1978, pp. 35-40.

[12] R. V. Plykin, Sources and Sinks of A-Diffeomorphisms, Math. Sbornik, Vol. 94 (136) (2), 1974, pp. 233-253.

[13] J. Palis and F. TAKens, Stability of parametrized families of gradient vector fields, Ann. of Math., Vol. 118, 1983, pp. 383-421.

[14] J. PALIS and F. TAKens, Hyperbolicity and sensitive chaotic dynamics at homoclinic bifurcations, Cambridge University Press, Vol. 35, 1993.

[15] A. Rovella, A Dinâmica das perturbações do Atrator de Lorenz Contrativo, These I.M.P.A., serie F-053-Julho/92.

[16] S. Smale, Differentiable dynamical systems, Bull. Am. Math. Soc., Vol. 73, 1967, pp. 747817.

[17] J. Sotomayor, $\Omega$-Explosion near saddle-node fixed point, Com. Anais Ac. B. Cienc., Vol. 41, No 4, pp. 644 R.1969.

[18] J. SотомаYoR, Generic bifurcations of dynamical systems, Dynamical Systems ed. M. M. Peixoto, Acad. Press, 1973, New York.

[19] F. TAKENS, Partially hyperbolic fixed points, Topology, Vol. 10, 1971, pp. 133-147.

[20] R. F. Williams, One dimensional non-wandering set, Topology, Vol. 6, 1969, pp. 473-487.

(Manuscript received December 16, 1994.) 